Pacific

Journal of

Mathematics

\author{
MINIMAL SURFACES WITH TWO ENDS \\ WHICH HAVE THE LEAST TOTAL ABSOLUTE CURVATURE \\ SHOICHI FUJIMORI AND TOSHIHIRO SHODA
}

Volume $282 \quad$ No. 1

May 2016 


\title{
MINIMAL SURFACES WITH TWO ENDS WHICH HAVE THE LEAST TOTAL ABSOLUTE CURVATURE
}

\author{
SHOICHI FUJIMORI AND TOSHIHIRO SHODA \\ Dedicated to Professor Reiko Miyaoka on the occasion of her retirement
}

\begin{abstract}
We consider complete noncatenoidal minimal surfaces of finite total curvature with two ends. A family of such minimal surfaces with least total absolute curvature is given. We also obtain a uniqueness theorem for this family from its symmetries.
\end{abstract}

\section{Introduction}

For a complete minimal surface in Euclidean space, an inequality stronger than the classical inequality of Cohn-Vossen holds, giving a lower bound for the total absolute curvature. It is then natural to ask whether there is a minimal surface which attains this minimum value for the total absolute curvature. We consider this problem and contribute to the theory of existence of minimal surfaces in Euclidean space. Our work connects with the Björling problem for minimal surfaces in Euclidean space.

Let $f: M \rightarrow \mathbb{R}^{3}$ be a minimal immersion of a 2-manifold $M$ into Euclidean 3 -space $\mathbb{R}^{3}$. We usually call $f$ a minimal surface in $\mathbb{R}^{3}$. Choosing isothermal coordinates makes $M$ a Riemann surface, and then $f$ is called a conformal minimal immersion. The following representation formula is one of the basic tools in the theory of minimal surfaces.

Theorem 1.1 (Weierstrass representation [Osserman 1964]). Let $(g, \eta)$ be a pair of a meromorphic function $g$ and a holomorphic differential $\eta$ on a Riemann surface $M$ such that

$$
\left(1+|g|^{2}\right)^{2} \eta \bar{\eta}
$$

Fujimori partially supported by JSPS Grant-in-Aid for Young Scientists (B) 25800047.

Shoda partially supported by JSPS Grant-in-Aid for Young Scientists (B) 24740047.

MSC2010: primary 53A10; secondary 49Q05, 53C42.

Keywords: minimal surface, finite total curvature, two ends. 
gives a Riemannian metric on $M$. We set

$$
\Phi:=\left(\begin{array}{c}
\left(1-g^{2}\right) \eta \\
i\left(1+g^{2}\right) \eta \\
2 g \eta
\end{array}\right)
$$

where $i=\sqrt{-1}$. Assume that

$$
\operatorname{Re} \oint_{\ell} \Phi=\mathbf{0} \text { holds for any } \ell \in \pi_{1}(M) .
$$

Then

$$
f=\operatorname{Re} \int_{z_{0}}^{z} \Phi: M \rightarrow \mathbb{R}^{3} \quad\left(z_{0} \in M\right)
$$

defines a conformal minimal immersion.

The pair $(g, \eta)$ in Theorem 1.1 is called the Weierstrass data of $f$.

Remark 1.2. The period condition $(\mathrm{P})$ of the minimal surface is equivalent to

$$
\oint_{\ell} \eta=\overline{\oint_{\ell} g^{2} \eta}
$$

and

$$
\operatorname{Re} \oint_{\ell} g \eta=0
$$

for any $\ell \in \pi_{1}(M)$.

Remark 1.3. The first fundamental form $d s^{2}$ and the second fundamental form $\mathbb{I}$ of the surface (1-3) are given by

$$
d s^{2}=\left(1+|g|^{2}\right)^{2} \eta \bar{\eta}, \quad \mathbb{I}=-\eta d g-\overline{\eta d g} .
$$

Moreover, $g: M \rightarrow \mathbb{C} \cup\{\infty\}$ coincides with the composition of the Gauss map $G: M \rightarrow S^{2}$ of the minimal surface and stereographic projection $\sigma: S^{2} \rightarrow \mathbb{C} \cup\{\infty\}$, that is, $g=\sigma \circ G$. So we call $g$ the Gauss map of the minimal surface.

Next, we assume that a minimal surface is complete and of finite total curvature. These two conditions give rise to restrictions on the topological and conformal types of minimal surfaces.

Theorem 1.4 [Huber 1957; Osserman 1964]. Let $f: M \rightarrow \mathbb{R}^{3}$ be a conformal minimal immersion. Suppose that $f$ is complete and of finite total curvature.

(1) $M$ is conformally equivalent to a compact Riemann surface $\bar{M}_{\gamma}$ of genus $\gamma$ punctured at a finite number of points $p_{1}, \ldots, p_{n}$.

(2) The Gauss map g extends to a holomorphic mapping $\hat{g}: \bar{M}_{\gamma} \rightarrow \mathbb{C} \cup\{\infty\}$.

The removed points $p_{1}, \ldots, p_{n}$ correspond to ends of the minimal surface. 
The asymptotic behavior around each end $p_{i}$ can be described by the order of the poles of $\Phi=\left(\Phi_{1}, \Phi_{2}, \Phi_{3}\right)$ in Theorem 1.1 at $p_{i}$. Let

$$
d_{i}=\max _{1 \leq j \leq 3}\left\{\operatorname{ord}\left(\Phi_{j}, p_{i}\right)\right\}-1,
$$

where $\operatorname{ord}\left(\Phi_{j}, p_{i}\right)$ is the order of the pole of $\Phi_{j}$ at $p_{i}(1 \leq i \leq n, 1 \leq j \leq 3)$. Condition (P) yields residue $\left(\Phi, p_{i}\right) \in \mathbb{R}^{3}$, and thus $d_{i} \geq 1$. The following theorem shows the geometric properties of $d_{i}$, which includes a stronger inequality than the Cohn-Vossen inequality.

Theorem 1.5 [Osserman 1964; Jorge and Meeks 1983; Schoen 1983]. Let the immersion $f: M \rightarrow \mathbb{R}^{3}$ be a minimal surface as in Theorem 1.4.

(a) The immersion $f$ is proper.

(b) If $S^{2}(r)$ is the sphere of radius $r$, then $\frac{1}{r}\left(f(M) \cap S^{2}(r)\right)$ consists of $n$ closed curves $\Gamma_{1}, \ldots, \Gamma_{n}$ in $S^{2}(1)$ which converge $C^{1}$ to closed geodesics $\gamma_{1}, \ldots, \gamma_{n}$ of $S^{2}(1)$, with multiplicities $d_{1}, \ldots, d_{n}$, as $r \rightarrow \infty$. Moreover,

$$
\begin{aligned}
\frac{1}{2 \pi} \int_{M} K d A & =\chi\left(\bar{M}_{\gamma}\right)-\sum_{i=1}^{n}\left(d_{i}+1\right) \\
& \leq \chi(M)-n=\chi\left(\bar{M}_{\gamma}\right)-2 n=2(1-\gamma-n),
\end{aligned}
$$

and equality holds if and only if each end is embedded.

The equation in the first line of (1-7) is called the Jorge-Meeks formula.

Moreover, a relation between the total (absolute) curvature and the degree of $g$ is as follows. Note that since $g$ extends to a holomorphic map $\hat{g}$ from a compact Riemann surface $\bar{M}_{\gamma}$ to a compact Riemann surface $\mathbb{C} \cup\{\infty\}$, we can define the degree of $g$ by $\operatorname{deg}(g):=\operatorname{deg}(\hat{g})$. Since the Gaussian curvature of a minimal surface $M \rightarrow \mathbb{R}^{3}$ is always nonpositive, its total absolute curvature $\tau(M):=\int_{M}|K| d A$ is given by

$$
\tau(M)=\int_{M}(-K) d A .
$$

Recall that the total absolute curvature of a minimal surface in $\mathbb{R}^{3}$ is just the area under the Gauss map $g: M \rightarrow \mathbb{C} \cup\{\infty\} \cong S^{2}$, that is,

$$
\tau(M)=\left(\text { the area of } S^{2}\right) \operatorname{deg}(g)=4 \pi \operatorname{deg}(g) \in 4 \pi \mathbb{Z} .
$$

(See, for example, (3.11) in [Hoffman and Osserman 1980] for details.) Hence (1-7) is rewritten as

$$
\operatorname{deg}(g) \geq \gamma+n-1
$$

and we consider sharpness of the inequality (1-8). 


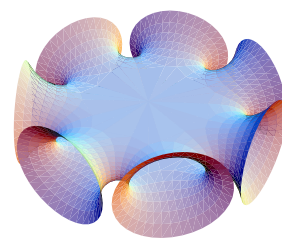

$(\gamma, n)=(0,7)$

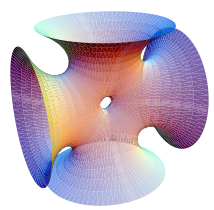

$(\gamma, n)=(1,4)$

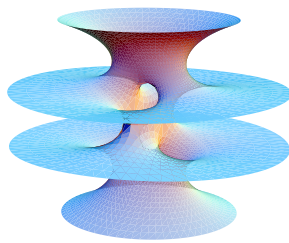

$(\gamma, n)=(2,4)$

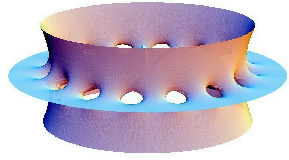

$(\gamma, n)=(14,3)$

Figure 1. Minimal surfaces with $n \geq 3$ satisfying equality in (1-8). For details on these surfaces, see [Jorge and Meeks 1983; Berglund and Rossman 1995; Wohlgemuth 1997; Hoffman and Meeks 1990], for instance.

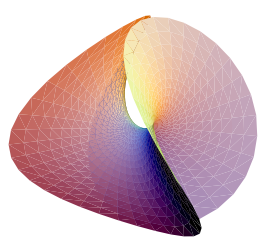

$\gamma=0$

Enneper

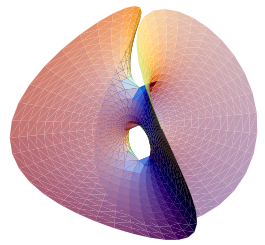

$\gamma=1$

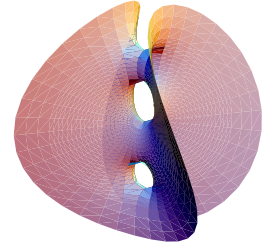

$\gamma=2$

Chen-Gackstatter

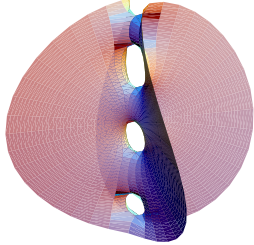

$\gamma=3$

Espírito Santo

Figure 2. Minimal surfaces with $n=1$ satisfying $\operatorname{deg}(g)=\gamma+1$.

For $n \geq 3$, there exist many examples of minimal surfaces which satisfy equality in $(1-8), \operatorname{deg}(g)=\gamma+n-1$. (See Figure 1.)

If $n=1$, then a minimal surface satisfying $\operatorname{deg}(g)=\gamma$ must be a plane. (See [Hoffman and Karcher 1997, Remark 2.2], for instance.) Thus on a nonplanar minimal surface, $\operatorname{deg}(g) \geq \gamma+1$. The existence of minimal surfaces with $\operatorname{deg}(g)=$ $\gamma+1$ was shown by C. C. Chen and F. Gackstatter [1982] (for $\gamma=1,2$ ), N. do Espírito Santo [1994] (for $\gamma=3$ ), K. Sato [1996], and M. Weber and M. Wolf [1998]. (See Figure 2.)

Finally, we consider the case $n=2$. In this case, the following uniqueness result is known.

Theorem 1.6 [Schoen 1983]. Let $f: M \rightarrow \mathbb{R}^{3}$ be a complete conformal minimal surface of finite total curvature. If $f$ has two ends and equality holds in (1-8), then $f$ must be a catenoid.

It follows that on a noncatenoidal minimal surface with two ends,

$$
\operatorname{deg}(g) \geq \gamma+2
$$

As a consequence, it is reasonable to consider: 
Problem 1.7. For an arbitrary genus $\gamma$, does there exist a complete conformal minimal surface of finite total curvature with two ends satisfying equality in (1-9)?

In the case $\gamma=0$, such minimal surfaces exist, and moreover, these minimal surfaces have been classified by F. J. López [1992]. (See Figure 3.) However, for the case $\gamma>0$, no answer to Problem 1.7 is known. Our first main result is to give a partial answer to this problem.

Main Theorem 1. If $\gamma$ is equal to 1 or an even number, there exists a complete conformal minimal surface of finite total curvature with two ends which satisfies equality in (1-9).

Note that if we do not assume the equality in (1-9), then there exists a complete conformal minimal surface of finite total curvature with two ends for an arbitrary genus $\gamma \geq 0$. (See [Fujimori and Shoda 2014], for instance.)

We prove Main Theorem 1 by explicit constructions in Section 2. We now discuss the asymptotic behavior for our minimal surfaces in terms of $d_{i}$. For a minimal surface as in Problem 1.7, we have $\left(d_{1}, d_{2}\right)=(1,3)$ or $(2,2)$. The case $\left(d_{1}, d_{2}\right)=(1,3)$ corresponds to a minimal surface with an embedded end and an Enneper type end. Recall that an embedded end is asymptotic to a plane or a catenoid (see [Schoen 1983]). The minimal surface given in Section 2A has an embedded end which is asymptotic to a plane and $\left(d_{1}, d_{2}\right)=(1,3)$ (see Corollary 2.3). The minimal surface introduced in Section 4A is another example with an embedded end which is asymptotic to a half catenoid and $\left(d_{1}, d_{2}\right)=(1,3)$. The minimal surfaces with $\left(d_{1}, d_{2}\right)=(2,2)$ are obtained in Section 2B (see Corollary 2.6).

The minimal surfaces given in Corollary 2.3 and Corollary 2.6 have symmetry groups with $4(\gamma+1)$ elements. Next we consider the uniqueness theorem for the symmetries. Uniqueness is also one of the important problems for minimal surfaces, and there are many uniqueness theorems (see [Martín and Weber 2001; Hoffman and Meeks 1990]). Our other main theorem is as follows.
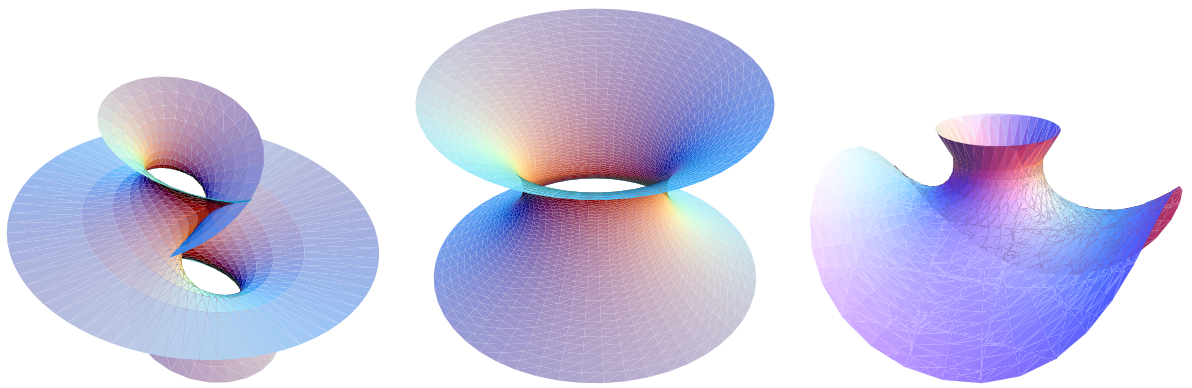

Figure 3. Examples for $\gamma=0$. The surface in the middle is a double cover of a catenoid. 
Main Theorem 2. Let $f: M \rightarrow \mathbb{R}^{3}$ be a complete conformal minimal surface of finite total curvature with two ends and genus $\gamma$. Suppose that $f$ satisfies equality in (1-9) and has $4(\gamma+1)$ symmetries. We assume either $\gamma=1$ and $\left(d_{1}, d_{2}\right)=(1,3)$, or $\gamma$ is an even number and $\left(d_{1}, d_{2}\right)=(2,2)$. Then $f$ is one of the minimal surfaces given in Main Theorem 1.

At the end of this section, we discuss our work from the point of view of the Björling problem for minimal surfaces. The classical Björling problem is to determine a piece of a minimal surface containing a given analytic strip. This was named after E. G. Björling in 1844. H. A. Schwarz gave an explicit solution to it. (See [Nitsche 1975], for instance.) Recently, Mira [2006] used the solution to the Björling problem to classify a certain class of minimal surfaces of genus 1. Also, Meeks and Weber [2007] produced an infinite sequence of complete minimal annuli by using the solution to the Björling problem and then gave a complete answer as to which curves appear as the singular set of a Colding-Minicozzi limit minimal lamination. Hence it is useful to study minimal surfaces from the point of view of the Björling problem. However, the existence of minimal surfaces of higher genus derived from the solution to the Björling problem seems to be unknown. In Section $2 \mathrm{~B}$, we show that our minimal surfaces, which have even numbers for the genus, are solutions to the Björling problem, and the generating curves are closed plane curves.

The paper is organized as follows: Section 2 contains constructions of concrete examples to prove Main Theorem 1, with the genus 1 case provided in Section 2A, and the even genus case in Section 2B. Section 2B also contains the result from the point of view of the Björling problem. We prove our uniqueness result in Section 3. In Section 4 we discuss remaining problems related to our work.

\section{Construction of surfaces for Main Theorem 1}

In this section we will construct the surfaces for proving Main Theorem 1. We will use the Weierstrass representation in Theorem 1.1, for which we need a Riemann surface $M$, a meromorphic function $g$, and a holomorphic differential $\eta$.

2A. The case $\gamma=\mathbf{1}$. Let $\bar{M}_{\gamma}$ be the Riemann surface

$$
\bar{M}_{\gamma}=\left\{(z, w) \in(\mathbb{C} \cup\{\infty\})^{2} \mid w^{\gamma+1}=z\left(z^{2}-1\right)^{\gamma}\right\} .
$$

The surface we will consider is

$$
M=\bar{M}_{\gamma} \backslash\{(0,0),(\infty, \infty)\},
$$

a Riemann surface of genus $\gamma$ from which two points have been removed. We want to define a complete conformal minimal immersion of $M$ into $\mathbb{R}^{3}$ by the Weierstrass 


\begin{tabular}{c|cccc}
$(z, w)$ & $(0,0)$ & $(1,0)$ & $(-1,0)$ & $(\infty, \infty)$ \\
\hline$g$ & $0^{1}$ & $0^{\gamma}$ & $0^{\gamma}$ & $\infty^{2 \gamma+1}$ \\
$\eta$ & $\infty^{\gamma+3}$ & & & $0^{3 \gamma+1}$ \\
\hline
\end{tabular}

Table 1. Orders of zeros and poles of $g$ and $\eta$.

representation in Theorem 1.1. To do this, set

$$
g=c w, \quad \eta=i \frac{d z}{z^{2} w},
$$

where $c \in \mathbb{R}_{>0}$ is a positive constant to be determined.

Let $\Phi$ be the $\mathbb{C}^{3}$-valued differential as in (1-2). We shall prove that (1-3) is a conformal minimal immersion of $M$.

We begin by showing by straightforward calculation how the following conformal diffeomorphisms $\kappa_{1}$ and $\kappa_{2}$ act on $\Phi$.

Lemma 2.1 (symmetries of the surface). Consider the conformal mappings

$$
\kappa_{1}(z, w)=(\bar{z}, \bar{w}), \quad \kappa_{2}(z, w)=\left(-z, e^{\pi i /(\gamma+1)} w\right)
$$

of $M$. Then

$$
\kappa_{1}^{*} \Phi=\left(\begin{array}{rrr}
-1 & 0 & 0 \\
0 & 1 & 0 \\
0 & 0 & -1
\end{array}\right) \Phi, \quad \kappa_{2}^{*} \Phi=\left(\begin{array}{ccc}
-\cos \frac{\pi}{\gamma+1} & \sin \frac{\pi}{\gamma+1} & 0 \\
-\sin \frac{\pi}{\gamma+1} & -\cos \frac{\pi}{\gamma+1} & 0 \\
0 & 0 & -1
\end{array}\right) \Phi .
$$

Since (1-1) gives a complete Riemannian metric on $M$ (see Table 1), it suffices to show that $f$ is well-defined on $M$ for the right choice of $c$.

Theorem 2.2. For any positive number $\gamma$, there exists a unique positive constant $c \in \mathbb{R}_{>0}$ for which the immersion $f$ given in (1-3) is well-defined on $M$.

Proof. To establish this theorem we must show (P) in Theorem 1.1. We will prove (1-4) and (1-5). But (1-5) follows from the exactness of $g \eta=i c d z / z^{2}=d(-i c / z)$, and thus we will only have to show (1-4). We first check the residues of $\eta$ and $g^{2} \eta$ at the ends $(0,0),(\infty, \infty)$. At the end $(0,0), w$ is a local coordinate for the Riemann surface $\bar{M}_{\gamma}$, and then $z=z(w)=w^{\gamma+1}\left\{(-1)^{\gamma}+\mathcal{O}\left(w^{2 \gamma+2}\right)\right\}$. We have

$$
\eta=\left(\frac{\alpha_{1}}{w^{\gamma+3}}+\mathcal{O}\left(w^{\gamma-1}\right)\right) d w \quad \text { and } \quad g^{2} \eta=\left(\frac{\alpha_{2}}{w^{\gamma+1}}+\mathcal{O}\left(w^{\gamma+1}\right)\right) d w
$$

where $\alpha_{j} \in \mathbb{C}$ (for $j=1,2$ ) are constants. These imply that both $\eta$ and $g^{2} \eta$ have no residues at $(0,0)$. Then the residue theorem yields that they have no residues at $(\infty, \infty)$ as well.

We next consider path-integrals along topological 1-cycles on $\bar{M}_{\gamma}$. We will give a convenient 1-cycle. 


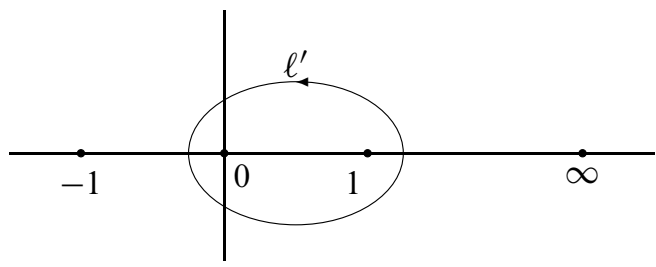

Figure 4. Projection to the $z$-plane of the loop $\ell^{\prime} \in \pi_{1}(M)$.

Define a 1-cycle on $\bar{M}_{\gamma}$ by

$$
\begin{aligned}
& \ell=\left\{(z, w)=\left(-t, \sqrt[\gamma+1]{-t\left(1-t^{2}\right)^{\gamma}} e^{\gamma \pi i /(\gamma+1)}\right) \mid-1 \leq t \leq 0\right\} \\
& \cup\left\{(z, w)=\left(t, \sqrt[\gamma+1]{t\left(1-t^{2}\right)^{\gamma}} e^{-\gamma \pi i /(\gamma+1)}\right) \mid 0 \leq t \leq 1\right\} .
\end{aligned}
$$

Recall that $(0,0)$ corresponds to the end of $f$. Avoiding the end $(0,0)$, we can deform $\ell$ to a 1-cycle $\ell^{\prime}$ on $M$ which is projected to a loop winding once around $[0,1]$ in the $z$-plane. (See Figure 4.)

By the actions of the $\kappa_{j}$ 's, we can obtain all of the 1-cycles on $M$ from $\ell^{\prime}$. If (P) holds for this $\ell^{\prime}$, then

$$
\operatorname{Re} \int_{\kappa_{j} \circ \ell^{\prime}} \Phi=\operatorname{Re} \int_{\ell^{\prime}} \kappa_{j}^{*} \Phi=K \operatorname{Re} \int_{\ell^{\prime}} \Phi=\mathbf{0}
$$

for some orthogonal matrix $K$, by Lemma 2.1. Hence all that remains to be done is to show that (1-4) holds for $\ell^{\prime}$.

We now calculate path-integrals of $\eta$ and $g^{2} \eta$ along $\ell^{\prime}$, and we want to reduce them to path-integrals along $\ell$ for simplicity. Note that both $\eta$ and $g^{2} \eta$ have poles at $(0,0)$. To avoid divergent integrals, here we add exact 1 -forms which have principal parts of $\eta$ and $g^{2} \eta$, respectively. It is straightforward to check

$$
\frac{d z}{z^{2} w}-\frac{\gamma+1}{\gamma+2} d\left(\frac{z^{2}-1}{z w}\right)=\frac{\gamma}{\gamma+2} \frac{d z}{w}, \quad \frac{w}{z^{2}} d z+\frac{\gamma+1}{\gamma} d\left(\frac{w}{z}\right)=\frac{2 w}{z^{2}-1} d z .
$$

So we have

$$
\begin{gathered}
\oint_{\ell^{\prime}} \eta=\frac{i \gamma}{\gamma+2} \oint_{\ell^{\prime}} \frac{d z}{w}=\frac{i \gamma}{\gamma+2} \oint_{\ell} \frac{d z}{w}=\frac{-2 \gamma}{\gamma+2} \sin \frac{\gamma \pi}{\gamma+1} \int_{0}^{1} \frac{d t}{\sqrt[\gamma+1]{t\left(1-t^{2}\right)^{\gamma}}} \\
\oint_{\ell^{\prime}} g^{2} \eta=2 i c^{2} \oint_{\ell^{\prime}} \frac{w}{z^{2}-1} d z=2 i c^{2} \oint_{\ell} \frac{w}{z^{2}-1} d z=-4 c^{2} \sin \frac{\gamma \pi}{\gamma+1} \int_{0}^{1} \sqrt[\gamma+1]{\frac{t}{1-t^{2}}} d t .
\end{gathered}
$$

By setting

$$
A_{\gamma}=\frac{\gamma}{\gamma+2} \int_{0}^{1} \frac{d t}{\sqrt[\gamma+1]{t\left(1-t^{2}\right)^{\gamma}}} \in \mathbb{R}_{>0}, \quad B_{\gamma}=2 \int_{0}^{1} \sqrt[\gamma+1]{\frac{t}{1-t^{2}}} d t \in \mathbb{R}_{>0}
$$




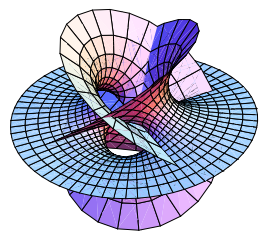

$\gamma=1$

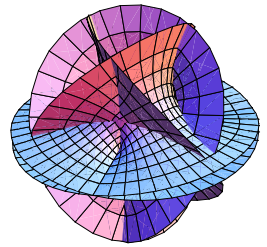

$\gamma=2$

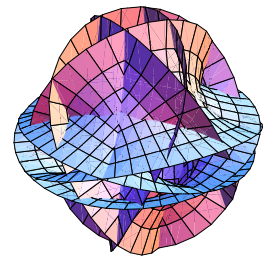

$\gamma=3$

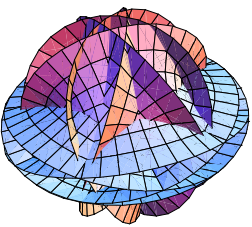

$\gamma=4$

Figure 5. Minimal surfaces of genus $\gamma$ with two ends which satisfy $\operatorname{deg}(g)=2 \gamma+1$.

the equation (1-4) is reduced to $A_{\gamma}=c^{2} B_{\gamma}$. Let us set

$$
c=\sqrt{\frac{A_{\gamma}}{B_{\gamma}}} \in \mathbb{R}_{>0} .
$$

This choice of $c$ satisfies (1-4) and is the unique positive real number that does so. This completes the proof. (See Figure 5.)

Since $\operatorname{deg}(g)=2 \gamma+1, \operatorname{deg}(g)=\gamma+2$ if and only if $\gamma=1$. As a consequence, the next corollary follows:

Corollary 2.3. There exists a complete conformal minimal surface of genus 1 with two ends which has least total absolute curvature.

2B. The case $\boldsymbol{\gamma}$ is even. The following construction is similar to the construction in Section 2A. Crucial arguments are given after (2-3).

For an integer $k \geq 2$, let $\bar{M}_{\gamma}$ be the Riemann surface

$$
\bar{M}_{\gamma}=\left\{(z, w) \in(\mathbb{C} \cup\{\infty\})^{2} \mid w^{k+1}=z^{2}\left(\frac{z-1}{z-a}\right)^{k}\right\},
$$

where $a \in(1, \infty)$ is a constant to be determined. By the Riemann-Hurwitz formula, we see that

$$
\gamma= \begin{cases}k & \text { if } k \text { is even, } \\ k-1 & \text { if } k \text { is odd }\end{cases}
$$

Note that the genus $\gamma$ is always even. (See Figure 6.)

We set

$$
\begin{aligned}
M & =\bar{M}_{\gamma} \backslash\{(0,0),(\infty, \infty)\}, \\
g & =c w \quad \text { for } c=a^{(k-2) /(2 k+2)} \in \mathbb{R}_{>0}, \\
\eta & =\frac{d z}{z w} .
\end{aligned}
$$

Then (1-1) gives a complete Riemannian metric $M$. (See Table 2.)

Also, $\operatorname{deg}(g)=k+2$ for all $k \geq 2$. Thus equality in (1-9) holds if and only if $k$ is even. Hereafter we assume $k$ is even. 
$k=2$
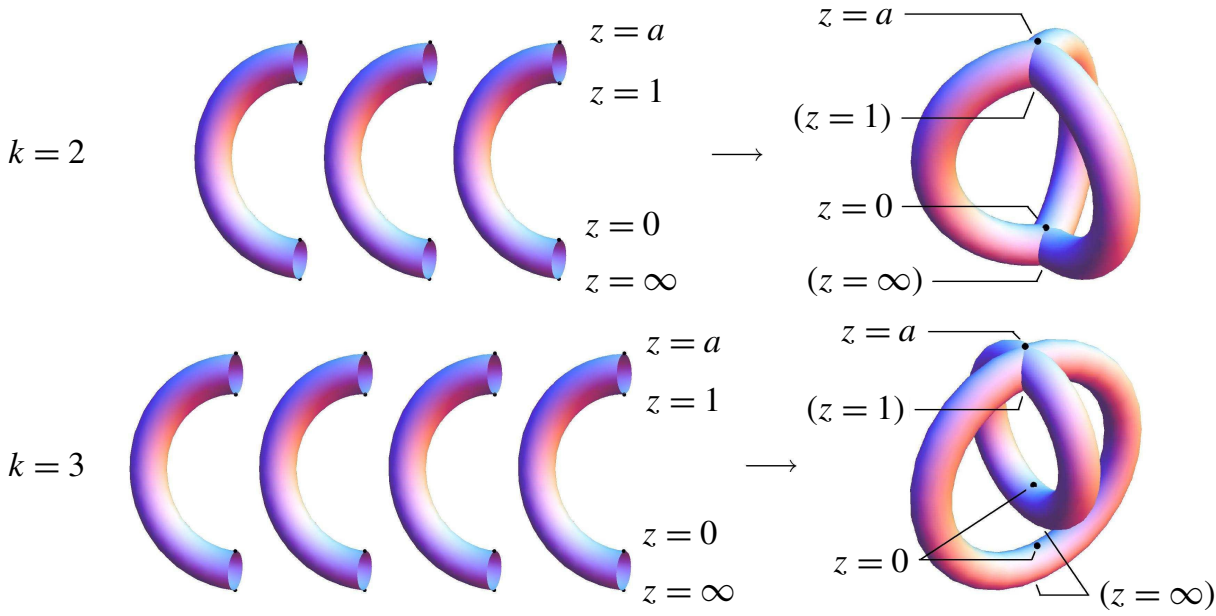

Figure 6. Riemann surfaces $\bar{M}_{\gamma}$ for $k=2$ (top) and $k=3$ (bottom). Both surfaces have genus 2 . When $k$ is odd, $\bar{M}_{\gamma}$ has self-intersections at $z=0$ and $z=\infty$. In the sketch in the bottom row, we see two different $z=0$ (and two different $z=\infty$, which are hidden from this viewpoint) but they are in fact the same points. The reason we place these points differently is to reveal their genus clearly.

\begin{tabular}{c|cccc}
$(z, w)$ & $(0,0)$ & $(1,0)$ & $(a, \infty)$ & $(\infty, \infty)$ \\
\hline$g$ & $0^{2}$ & $0^{k}$ & $\infty^{k}$ & $\infty^{2}$ \\
$\eta$ & $\infty^{4}$ & & $0^{2 k}$ & \\
\hline & & & & \\
$(z, w)$ & $(0,0)$ & $(1,0)$ & $(a, \infty)$ & $(\infty, \infty)$ \\
\hline$g$ & $0^{2}$ & $0^{k}$ & $\infty^{k}$ & $\infty^{2}$ \\
$\eta$ & $\infty^{3}$ & & $0^{2 k}$ & $0^{1}$ \\
\hline
\end{tabular}

Table 2. Orders of zeros and poles of $g$ and $\eta$ when $k$ is odd (top) and $k$ is even (bottom).

Let $\Phi$ be the $\mathbb{C}^{3}$-valued differential as in (1-2). We shall prove that (1-3) is a conformal minimal immersion of $M$.

First, we observe the following symmetries $\kappa_{1}, \kappa_{2}$, and $\kappa_{3}$ of the surface.

Lemma 2.4 (Symmetries of the surface). Consider the conformal mappings

$$
\kappa_{1}(z, w)=(\bar{z}, \bar{w}), \quad \kappa_{2}(z, w)=\left(z, e^{2 \pi i /(k+1)} w\right), \quad \kappa_{3}(z, w)=\left(\frac{a}{z}, \frac{1}{c^{2} w}\right)
$$


of $M$. Then

$$
\begin{gathered}
\kappa_{1}^{*} \Phi=\left(\begin{array}{rrr}
1 & 0 & 0 \\
0 & -1 & 0 \\
0 & 0 & 1
\end{array}\right) \Phi, \quad \kappa_{2}^{*} \Phi=\left(\begin{array}{ccc}
\cos \frac{2 \pi}{k+1} & -\sin \frac{2 \pi}{k+1} & 0 \\
\sin \frac{2 \pi}{k+1} & \cos \frac{2 \pi}{k+1} & 0 \\
0 & 0 & 1
\end{array}\right) \Phi, \\
\kappa_{3}^{*} \Phi=\left(\begin{array}{rrr}
1 & 0 & 0 \\
0 & -1 & 0 \\
0 & 0 & -1
\end{array}\right) \Phi .
\end{gathered}
$$

As we have already seen the completeness of $f$, it suffices to show that $f$ is well-defined on $M$ for the right choice of $a \in(1, \infty)$.

Theorem 2.5. For any positive even number $k$, there exists a unique constant $a \in(1, \infty)$ for which the immersion $f$ given in (1-3) is well-defined on $M$.

Proof. We will show $(\mathrm{P})$ in Theorem 1.1. It is easy to verify that there are no residues at the ends $(0,0)$ and $(\infty, \infty)$. So all that remains is to choose $c$ so that (P) is satisfied. The equation (1-5) follows from the exactness of

$$
g \eta=\frac{c}{z} d z=c \cdot d(\log z)
$$

and $c \in \mathbb{R}$, and hence we will only have to show (1-4). To do this, we will give convenient 1-cycles.

Define a 1-cycle on $\bar{M}_{\gamma}$ as

$$
\begin{aligned}
\ell_{1}=\left\{(z, w)=\left(-t, \sqrt[k+1]{t^{2}\left(\frac{1+t}{a+t}\right)^{k}}\right) \mid-1 \leq t \leq 0\right\} \\
\cup\left\{(z, w)=\left(t, \sqrt[k+1]{t^{2}\left(\frac{1-t}{a-t}\right)^{k}} e^{2 \pi i /(k+1)}\right) \mid 0 \leq t \leq 1\right\} .
\end{aligned}
$$

Recall that $(0,0)$ corresponds to the end of $f$. Avoiding the end $(0,0)$, we can deform $\ell_{1}$ to a 1-cycle $\ell_{1}^{\prime}$ on $M$ which is projected to a loop winding once around $[0,1]$ in the $z$-plane. We also define another 1-cycle on $M$ as

$$
\begin{aligned}
\ell_{2}=\left\{(z, w)=\left(-t, \sqrt[k+1]{t^{2}\left(\frac{-t-1}{a+t}\right)^{k}} e^{k \pi i /(k+1)}\right) \mid-a \leq t \leq-1\right\} \\
\cup\left\{(z, w)=\left(t, \sqrt[k+1]{t^{2}\left(\frac{t-1}{a-t}\right)^{k}} e^{-k \pi i /(k+1)}\right) \mid 1 \leq t \leq a\right\} .
\end{aligned}
$$

(See Figure 7.)

Again by the actions of the $\kappa_{j}$ 's, we can obtain all of the 1-cycles on $M$ from $\ell_{1}^{\prime}$ and $\ell_{2}$. We now show that (1-4) holds for $\ell_{1}^{\prime}$ and $\ell_{2}$. 


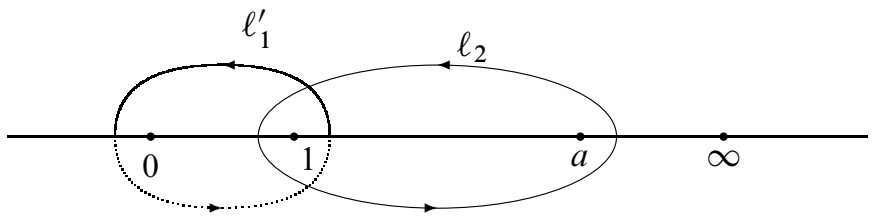

Figure 7. Projections to the $z$-plane of the loops $\ell_{1}^{\prime}$ and $\ell_{2} \in \pi_{1}(M)$.

First we calculate the path-integrals of $\eta$ and $g^{2} \eta$ along $\ell_{2}$. Then we have

$$
\begin{aligned}
\oint_{\ell_{2}} \eta & =2 i \sin \frac{k \pi}{k+1} \int_{1}^{a} \sqrt[k+1]{\frac{(a-t)^{k}}{t^{k+3}(t-1)^{k}}} d t, \\
\oint_{\ell_{2}} g^{2} \eta & =-2 i c^{2} \sin \frac{k \pi}{k+1} \int_{1}^{a} \sqrt[k+1]{\frac{(t-1)^{k}}{t^{k-1}(a-t)^{k}}} d t \\
& =-2 i \sin \frac{k \pi}{k+1} \int_{1}^{a} \sqrt[k+1]{\frac{(a-\tau)^{k}}{\tau^{k+3}(\tau-1)^{k}}} d \tau,
\end{aligned}
$$

where $\tau=a / t$. As a result, (1-4) holds for $\ell_{2}$.

Next we calculate the path-integrals of $\eta$ and $g^{2} \eta$ along $\ell_{1}^{\prime}$, and we want to reduce them to the path-integrals along $\ell_{1}$. Note that $\eta$ has a pole at $(0,0)$. To avoid a divergent integral, here we add an exact 1-form which has the principal part of $\eta$. It is straightforward to check

$$
\eta-\frac{k+1}{2} d\left(\frac{z-1}{w}\right)=-\frac{k}{2} \frac{z-1}{w(z-a)} d z+\frac{1}{2} \frac{d z}{w} .
$$

Thus we have

$$
\begin{aligned}
\oint_{\ell_{1}^{\prime}} \eta & =\oint_{\ell_{1}^{\prime}}\left(-\frac{k}{2} \frac{z-1}{w(z-a)} d z+\frac{1}{2} \frac{d z}{w}\right) \\
& =\oint_{\ell_{1}}\left(-\frac{k}{2} \frac{z-1}{w(z-a)} d z+\frac{1}{2} \frac{d z}{w}\right)=i e^{-\pi i /(k+1)} \sin \frac{\pi}{k+1}\left(k A_{1}-A_{2}\right),
\end{aligned}
$$

where

$$
\begin{aligned}
& A_{1}=\int_{0}^{1} \frac{(1-t)^{1 /(k+1)}}{t^{2 /(k+1)}(a-t)^{1 /(k+1)}} d t, \\
& A_{2}=\int_{0}^{1} \frac{(a-t)^{k /(k+1)}}{t^{2 /(k+1)}(1-t)^{k /(k+1)}} d t .
\end{aligned}
$$

Also, we have

$$
\oint_{\ell_{1}^{\prime}} g^{2} \eta=\oint_{\ell_{1}} g^{2} \eta=2 i e^{\pi i /(k+1)} \sin \frac{\pi}{k+1} a^{(k-2) /(k+1)} A_{3},
$$


where

$$
A_{3}=\int_{0}^{1} \frac{(1-t)^{k /(k+1)}}{t^{(k-1) /(k+1)}(a-t)^{k /(k+1)}} d t .
$$

Hence for the loop $\ell_{1}^{\prime} \in \pi_{1}(M)$, (1-4) holds if and only if

$$
k A_{1}+2 a^{(k-2) /(k+1)} A_{3}-A_{2}=0 .
$$

Now we evaluate the values $A_{1}, A_{2}$, and $A_{3}$. Since $1 / a \leq 1 /(a-t) \leq 1 /(a-1)$, we see that

$$
\begin{gathered}
\frac{1}{a^{1 /(k+1)}} B\left(\frac{k-1}{k+1}, \frac{k+2}{k+1}\right) \leq A_{1} \leq \frac{1}{(a-1)^{1 /(k+1)}} B\left(\frac{k-1}{k+1}, \frac{k+2}{k+1}\right), \\
\frac{1}{a^{k /(k+1)}} B\left(\frac{2}{k+1}, \frac{2 k+1}{k+1}\right) \leq A_{3} \leq \frac{1}{(a-1)^{k /(k+1)}} B\left(\frac{2}{k+1}, \frac{2 k+1}{k+1}\right),
\end{gathered}
$$

where $B(x, y)$ is the beta function defined by

$$
B(x, y)=\int_{0}^{1} t^{x-1}(1-t)^{y-1} d t \quad \text { for } \operatorname{Re} x>0 \text { and } \operatorname{Re} y>0 .
$$

Also, since $a-1 \leq a-t \leq a$, we have

$$
(a-1)^{k /(k+1)} B\left(\frac{k-1}{k+1}, \frac{1}{k+1}\right) \leq A_{2} \leq a^{k /(k+1)} B\left(\frac{k-1}{k+1}, \frac{1}{k+1}\right) .
$$

It follows that for the case $a \rightarrow \infty$, we have $A_{1} \rightarrow 0, a^{(k-2) /(k+1)} A_{3} \rightarrow 0$, and $A_{2} \rightarrow \infty$. As a result, the left-hand side of (2-3) is negative. On the other hand, for the case $a \rightarrow 1$, we have

$$
\begin{aligned}
& k A_{1}+2 a^{(k-2) /(k+1)} A_{3}-A_{2} \\
& \geq \frac{k}{a^{1 /(k+1)}} B\left(\frac{k-1}{k+1}, \frac{k+2}{k+1}\right)+\frac{2}{a^{2 /(k+1)}} B\left(\frac{2}{k+1}, \frac{2 k+1}{k+1}\right)-a^{k /(k+1)} B\left(\frac{k-1}{k+1}, \frac{1}{k+1}\right) \\
& =\frac{1}{a^{1 /(k+1)}} B\left(\frac{k-1}{k+1}, \frac{1}{k+1}\right)+\frac{2}{a^{2 /(k+1)}} B\left(\frac{2}{k+1}, \frac{2 k+1}{k+1}\right)-a^{k /(k+1)} B\left(\frac{k-1}{k+1}, \frac{1}{k+1}\right) \\
& \underset{a \rightarrow 1}{\longrightarrow} 2 B\left(\frac{2}{k+1}, \frac{2 k+1}{k+1}\right)>0,
\end{aligned}
$$

and here we use formula

$$
B(x, y+1)=\frac{y}{x+y} B(x, y)
$$

for the beta function. So, the left-hand side of (2-3) is positive.

Thus, the intermediate value theorem yields that there exists $a \in(1, \infty)$ which satisfies (2-3). Moreover, since each of $A_{1}, a^{(k-2) /(k+1)} A_{3}$, and $-A_{2}$ is a monotone decreasing function with respect to $a$, the left-hand side of (2-3) is a monotone decreasing function as well. This proves the uniqueness. (See Figure 8). 

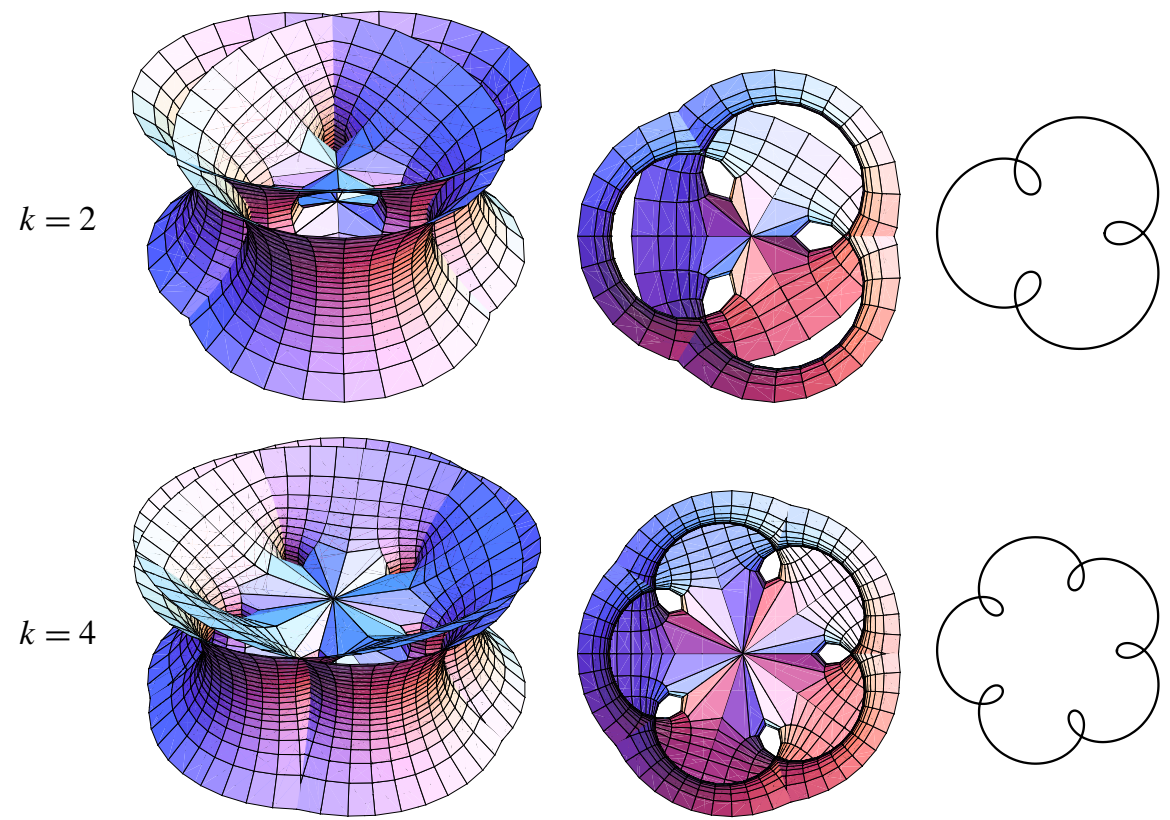

Figure 8. Minimal surfaces of genus $k$ with two ends which satisfy $\operatorname{deg}(g)=k+2$. The middle columns show a half cut away from the surfaces by the $x y$-plane. The right columns show their intersection with the $x y$-plane.

Since $\operatorname{deg}(g)=\gamma+2$, the next corollary follows.

Corollary 2.6. For all even numbers $\gamma$, there exists a complete conformal minimal surface of genus $\gamma$ with two ends which has least total absolute curvature.

Combining Corollaries 2.3 and 2.6 proves Main Theorem 1.

Next we discuss the above minimal surfaces from the point of view of the Björling problem. As we mentioned in the introduction, there is a construction method for minimal surfaces from a given curve. We show that every minimal surface given in this subsection gives a solution to the Björling problem in the higher genus case and the generating curve is a closed planar geodesic.

Let $l$ be a fixed point set of $\kappa_{3} \circ \kappa_{1}$. Using (1-1), we see that $\kappa_{3} \circ \kappa_{1}$ is an isometry, and thus $l$ is a geodesic. An explicit description of $l$ is given by

$$
\frac{a}{\bar{z}}=z, \quad \frac{1}{c^{2} \bar{w}}=w,
$$

that is,

$$
|z|=\sqrt{a}, \quad|w|=\frac{1}{c} .
$$


Hence we conclude that $l$ is a closed geodesic. Moreover, by Lemma 2.4, $l$ lies in the $x y$-plane, and therefore the assertion follows.

\section{Uniqueness}

In this section, we will prove Main Theorem 2 through four subsections.

3A. Symmetry. First, we refer to some basic results about symmetries of a minimal surface. (See p. 349 in [López and Martín 1999].)

Let $f: M \rightarrow \mathbb{R}^{3}$ be a conformal minimal immersion, with $(g, \eta)$ its Weierstrass data. Suppose that $A: M \rightarrow M$ is a diffeomorphism. $A$ is said to be a symmetry if there exists $O \in \mathcal{O}(3, \mathbb{R})$ and $v \in \mathbb{R}^{3}$ such that

$$
(f \circ A)(p)=O f(p)+v .
$$

Denote by $\operatorname{Sym}(M)$ the group of symmetries of $M$, and by $\operatorname{Iso}(M)$ the isometry group of $M$. Then, by definition, $\operatorname{Sym}(M)$ is a subgroup of $\operatorname{Iso}(M)$. Let $\mathrm{L}(M)$ be the group of holomorphic and antiholomorphic diffeomorphisms $\alpha$ of $M$ satisfying

$$
G \circ \alpha(p)=O \circ G(p),
$$

where $G: M \rightarrow S^{2}$ is the Gauss map and $O \in \mathcal{O}(3, \mathbb{R})$ is a linear isometry of $\mathbb{R}^{3}$. We now assume that $f$ is complete, and of finite total curvature. López and Martín pointed out that if one of the three differentials $\left(1-g^{2}\right) \eta, i\left(1+g^{2}\right) \eta$, and $2 g \eta$ is not exact, then

$$
\mathrm{L}(M)=\operatorname{Iso}(M)=\operatorname{Sym}(M) .
$$

Suppose that $f$ has two ends. By Theorem 1.4, there exists a compact Riemann surface $\bar{M}_{\gamma}$ of genus $\gamma$ and two points $p_{1}, p_{2} \in \bar{M}_{\gamma}$ such that $M$ is conformally equivalent to $\bar{M}_{\gamma}-\left\{p_{1}, p_{2}\right\}$. A symmetry of $f(M)$ extends to $\bar{M}_{\gamma}$ leaving the set $\left\{p_{1}, p_{2}\right\}$ invariant. By the Hurwitz theorem, the group $\operatorname{Sym}(M)$ is finite, and so up to a suitable choice of the origin, $\operatorname{Sym}(M)$ is a finite group $\Delta$ of orthogonal linear transformations of $\mathbb{R}^{3}$.

We assume that $\operatorname{Sym}(M)$ has $4(\gamma+1)$ elements (for $\gamma \geq 1)$ and also that $\mathrm{L}(M)=\operatorname{Iso}(M)=\operatorname{Sym}(M)$. If there is no symmetry in $\Delta$ such that either $p_{1}$ or $p_{2}$ is fixed, then $\Delta$ has at most 2 elements by a fundamental argument in linear algebra. Hence, we may assume without loss of generality that there exists a symmetry which fixes $p_{1}$. Up to rotations, we may assume $g\left(p_{1}\right)=0$, and then $\Delta$ leaves the $x_{3}$-axis invariant.

We now focus on the following two cases: the case $\gamma=1$ with $\left(d_{1}, d_{2}\right)=(1,3)$, and the even genus case with $\left(d_{1}, d_{2}\right)=(2,2)$ (for the definition of $d_{i}$, see (1-6)). For the former case, every symmetry in $\Delta$ leaves $p_{i}$ invariant. So we see $g\left(p_{2}\right)=0$ or $\infty$. For the latter case, we have $|\Delta| \geq 12$, and then there exist at least two symmetries which leave $p_{i}$ invariant. Hence $g\left(p_{2}\right)=0$ or $\infty$. 
Let $\Delta_{0}$ be the subgroup of holomorphic transformations in $\Delta$, and denote by $\mathcal{R} \subset \Delta_{0}$ the cyclic subgroup of rotations around the $x_{3}$-axis. Clearly, we obtain that

$$
\left[\Delta: \Delta_{0}\right] \leq 2, \quad\left[\Delta_{0}: \mathcal{R}\right] \leq 2 .
$$

So the subgroups $\Delta_{0} \subset \Delta$ and $\mathcal{R} \subset \Delta_{0}$ are both normal.

Let $R$ be the rotation around the $x_{3}$-axis with the smallest positive angle in $\Delta_{0}$, that is, $\mathcal{R}=\langle R\rangle$. We first consider the quotient map $\pi_{\mathcal{R}}: \bar{M}_{\gamma} \rightarrow \bar{M}_{\gamma} / \mathcal{R}$. From (3-1), we see that

$$
\operatorname{deg}\left(\pi_{\mathcal{R}}\right)=|\mathcal{R}| \geq \gamma+1 .
$$

By the Riemann-Hurwitz formula, we have

$$
\begin{aligned}
|\mathcal{R}|\left(2-2 \gamma\left(\bar{M}_{\gamma} / \mathcal{R}\right)\right) & =2-2 \gamma+\sum_{p \in \bar{M}_{\gamma}}(\mu(p)-1) \\
& =2-2 \gamma+2(|\mathcal{R}|-1)+\sum_{p \in M}(\mu(p)-1),
\end{aligned}
$$

where $\gamma\left(\bar{M}_{\gamma} / \mathcal{R}\right)$ is the genus of $\bar{M}_{\gamma} / \mathcal{R}$ and $\mu(p)-1$ is the ramification index at $p$. Let $q_{1}^{\prime}, \ldots, q_{t}^{\prime}$ be ramified values of $\pi_{\mathcal{R}}$ except for the $\pi_{\mathcal{R}}\left(p_{i}\right)$ 's, and $m_{i}-1$ the ramification index at $p \in \pi_{\mathcal{R}}^{-1}\left(q_{i}^{\prime}\right)$. Note that $2 \leq m_{i} \leq|\mathcal{R}|$ and the ramification index at $p_{i}$ is $|\mathcal{R}|-1$. Combining (3-2) and (3-3) yields

$$
\begin{aligned}
|\mathcal{R}|\left(2-2 \gamma\left(\bar{M}_{\gamma} / \mathcal{R}\right)\right) & =2-2 \gamma+2(|\mathcal{R}|-1)+\sum_{i=1}^{t}\left(m_{i}-1\right) \frac{|\mathcal{R}|}{m_{i}} \\
& \geq 2+\sum_{i=1}^{t}\left(m_{i}-1\right) \frac{|\mathcal{R}|}{m_{i}}>0 .
\end{aligned}
$$

It follows that $\gamma\left(\bar{M}_{\gamma} / \mathcal{R}\right)=0$. This, combined with (3-2) and (3-4), implies

$$
2 \gamma=\sum_{i=1}^{t}\left(m_{i}-1\right) \frac{|\mathcal{R}|}{m_{i}}=|\mathcal{R}|\left(\sum_{i=1}^{t}\left(\frac{1}{2}-\frac{1}{m_{i}}\right)+\frac{t}{2}\right) \geq(\gamma+1) \frac{t}{2} .
$$

So $t \leq 4 \gamma /(\gamma+1)<4$, and thus $t=1,2$, 3. We remark that we have $|\mathcal{R}|=\gamma+1$, $2(\gamma+1)$, and $4(\gamma+1)$.

The case $t=1$.

From the first equality of (3-5), we obtain $2 \gamma=|\mathcal{R}|\left(1-\frac{1}{m_{1}}\right)$. For the case $|\mathcal{R}|=\gamma+1$, we get $\frac{1}{m_{1}}=-\frac{\gamma-1}{\gamma+1} \leq 0$, which is absurd. Next, if $|\mathcal{R}|=2(\gamma+1)$ then $m_{1}=\gamma+1$ holds. Finally, $|\mathcal{R}|=4(\gamma+1)$ gives

$$
\frac{1}{2}>\frac{\gamma}{2(\gamma+1)}=1-\frac{1}{m_{1}} \geq 1-\frac{1}{2}=\frac{1}{2},
$$

which leads to a contradiction. 
The case $t=2$.

We obtain $2 \gamma=|\mathcal{R}|\left(2-\frac{1}{m_{1}}-\frac{1}{m_{2}}\right)$ from the first equality of (3-5). Without loss of generality, we may assume $m_{1} \leq m_{2}$. Then

$$
2-\frac{2}{m_{1}} \leq \frac{2 \gamma}{|\mathcal{R}|}=2-\frac{1}{m_{1}}-\frac{1}{m_{2}} \leq 2-\frac{2}{m_{2}} .
$$

For the case $|\mathcal{R}|=\gamma+1$, the inequality $\gamma+1 \leq m_{2} \leq|\mathcal{R}|=\gamma+1$ holds, and thus $m_{2}=\gamma+1$ and $m_{1}=\gamma+1$. Next we consider the case $|\mathcal{R}| \geq 2(\gamma+1)$. In this case, we have

$$
2-\frac{2}{m_{1}} \leq \frac{2 \gamma}{|\mathcal{R}|} \leq \frac{\gamma}{\gamma+1}<1
$$

and so $m_{1}<2$, which is absurd.

The case $t=3$.

It follows from the first equality of (3-5) that $2 \gamma=|\mathcal{R}|\left(3-\frac{1}{m_{1}}-\frac{1}{m_{2}}-\frac{1}{m_{3}}\right)$. We may assume $m_{1} \leq m_{2} \leq m_{3}$. Then

$$
3-\frac{3}{m_{1}} \leq \frac{2 \gamma}{|\mathcal{R}|}=3-\frac{1}{m_{1}}-\frac{1}{m_{2}}-\frac{1}{m_{3}} \leq 3-\frac{3}{m_{3}} .
$$

For the case $|\mathcal{R}|=\gamma+1$, we have $3-\frac{3}{m_{1}} \leq \frac{2 \gamma}{|\mathcal{R}|}=\frac{2 \gamma}{\gamma+1}<2$. So $m_{1}<3$, and hence $m_{1}=2$. As a result,

$$
\frac{5}{2}-\frac{2}{m_{2}} \leq \frac{2 \gamma}{\gamma+1}=\frac{5}{2}-\frac{1}{m_{2}}-\frac{1}{m_{3}} \leq \frac{5}{2}-\frac{2}{m_{3}} .
$$

The inequality $\frac{5}{2}-\frac{2}{m_{2}} \leq \frac{2 \gamma}{\gamma+1}$ gives $m_{2} \leq \frac{4(\gamma+1)}{\gamma+5}<4$. Thus $m_{2}=2$ or 3 . For the case $m_{2}=2$,

$$
\frac{2 \gamma}{\gamma+1}=2-\frac{1}{m_{3}}
$$

holds, and so $m_{3}=\frac{\gamma+1}{2}$. For the case $m_{2}=3$,

$$
\frac{2 \gamma}{\gamma+1}=\frac{13}{6}-\frac{1}{m_{3}} \text {. }
$$

It follows that $m_{3}=\frac{6(\gamma+1)}{\gamma+13}<6$, and hence $m_{3}=3,4$, or 5 . So $\gamma=11,23$, or 59 . For the case $|\mathcal{R}| \geq 2(\gamma+1)$,

$$
3-\frac{3}{m_{1}} \leq \frac{2 \gamma}{|\mathcal{R}|} \leq \frac{\gamma}{\gamma+1}<1
$$

So $m_{1}<\frac{3}{2}$, and this contradicts $m_{1} \geq 2$.

As a consequence, we obtain Tables 3, 4, and 5.

Note that, for $t=2, \pi_{\mathcal{R}}$ is a cyclic branched cover of $S^{2}$, of order $\gamma+1$, whose branch points are the fixed points of $\mathcal{R}$, namely $p_{1}, p_{2}, \pi_{\mathcal{R}}^{-1}\left(q_{1}^{\prime}\right), \pi_{\mathcal{R}}^{-1}\left(q_{2}^{\prime}\right)$.

If $t=1$, then $\pi_{\mathcal{R}}^{-1}\left(q_{1}^{\prime}\right)=\left\{q_{1}, q_{2}\right\}$ for some two points $q_{1}, q_{2} \in \bar{M}_{\gamma}$. If $R$ leaves every $q_{i}$ invariant, then $m_{1}$ must be $2(\gamma+1)$. Thus $R\left(q_{1}\right)=q_{2}$ and $R\left(q_{2}\right)=q_{1}$. 


\begin{tabular}{c|c}
$|\mathcal{R}|$ & $m_{i}$ \\
\hline$|\mathcal{R}|=\gamma+1$ & does not occur \\
$|\mathcal{R}|=2(\gamma+1)$ & $m_{1}=\gamma+1$ \\
$|\mathcal{R}|=4(\gamma+1)$ & does not occur \\
\hline
\end{tabular}

Table 3. The case $t=1$.

\begin{tabular}{c|c}
\multicolumn{1}{c|}{$|\mathcal{R}|$} & $m_{i}$ \\
\hline$|\mathcal{R}|=\gamma+1$ & $m_{1}=m_{2}=\gamma+1$ \\
$|\mathcal{R}| \geq 2(\gamma+1)$ & does not occur \\
\hline
\end{tabular}

Table 4. The case $t=2$.

\begin{tabular}{c|cccc}
\multicolumn{1}{c|}{$|\mathcal{R}|$} & \multicolumn{4}{|c}{$m_{i}$} \\
\hline \multirow{3}{*}{$|\mathcal{R}|=\gamma+1$} & $m_{1}$ & $m_{2}$ & $m_{3}$ & $\gamma$ \\
\cline { 2 - 5 } & 2 & 2 & $(\gamma+1) / 2$ & odd $(>1)$ \\
& 2 & 3 & 3 & 11 \\
& 2 & 3 & 4 & 23 \\
$|\mathcal{R}| \geq 2(\gamma+1)$ & 2 & 3 & 5 & 59 \\
\hline
\end{tabular}

Table 5. The case $t=3$.

So $R^{2}\left(q_{i}\right)=q_{i}$ and $f\left(q_{1}\right)=f\left(q_{2}\right) \in\left\{x_{3}\right.$-axis $\}$. Now we consider the quotient map $\pi_{\left\langle R^{2}\right\rangle}: \bar{M}_{\gamma} \rightarrow \bar{M}_{\gamma} /\left\langle R^{2}\right\rangle$. From the Riemann-Hurwitz formula,

$$
\begin{aligned}
\left|\left\langle R^{2}\right\rangle\right|\left(2-2 \gamma\left(\bar{M} /\left\langle R^{2}\right\rangle\right)\right) & =2-2 \gamma+4\left(\left|\left\langle R^{2}\right\rangle\right|-1\right) \\
& =2 \gamma+2>0 .
\end{aligned}
$$

Hence we obtain $\gamma\left(\bar{M} /\left\langle R^{2}\right\rangle\right)=0$. It follows that $\pi_{\left\langle R^{2}\right\rangle}$ is a cyclic branched cover of $S^{2}$, of order $\gamma+1$, whose branch points are $p_{1}, p_{2}, q_{1}, q_{2}$. This case corresponds to the case $t=2$, and thus we can determine the case $t=1$ after we consider the case $t=2$.

Next, we consider the quotient map $\pi_{\Delta_{0}}: \bar{M}_{\gamma} \rightarrow \bar{M}_{\gamma} / \Delta_{0}$ and repeat similar arguments as above. From the Riemann-Hurwitz formula, we obtain

$$
2 \gamma-2=\left|\Delta_{0}\right|\left(2 \gamma\left(\bar{M}_{\gamma} / \Delta_{0}\right)-2\right)+\sum_{p \in \bar{M}_{\gamma}}(\mu(p)-1) .
$$

We now treat the two cases that there is a symmetry $\sigma \in \Delta_{0}$ satisfying $\sigma\left(p_{1}\right)=p_{2}$ or not. For our case, we may exclude the case $t=3$, and consider the case $t=2$. It follows from (3-1) that $\left|\Delta_{0}\right|=2(\gamma+1)$ and $\sigma \in \Delta_{0} \backslash \mathcal{R}$. 
Case 1. The point $p_{1}$ can be transformed to $p_{2}$.

If there exists such $\sigma$, then the ramification index at $p_{i}$ must be $\left|\Delta_{0}\right| / 2-1$. So (3-6) can be reduced to

$$
2 \gamma-2=\left|\Delta_{0}\right|\left(2 \gamma\left(\bar{M} / \Delta_{0}\right)-2\right)+2\left(\left|\Delta_{0}\right| / 2-1\right)+\sum_{p \in M}(\mu(p)-1) .
$$

Hence,

$$
\begin{aligned}
2 \gamma & =\left|\Delta_{0}\right|\left(2 \gamma\left(\bar{M} / \Delta_{0}\right)-1\right)+\sum_{p \in M}(\mu(p)-1) \\
& =2(\gamma+1)\left(2 \gamma\left(\bar{M} / \Delta_{0}\right)-1\right)+\sum_{p \in M}(\mu(p)-1) \\
& \geq 2(\gamma+1)\left(2 \gamma\left(\bar{M} / \Delta_{0}\right)-1\right) .
\end{aligned}
$$

So the case $\gamma\left(\bar{M} / \Delta_{0}\right)>0$ leads to a contradiction, and thus $\gamma\left(\bar{M} / \Delta_{0}\right)=0$ holds. As a consequence, (3-7) can be reduced to

$$
4 \gamma+2=\sum_{p \in M}(\mu(p)-1) .
$$

Let $r_{1}^{\prime}, \ldots, r_{s}^{\prime}$ be ramified values of $\pi_{\Delta_{0}}$ except for the $\pi_{\Delta_{0}}\left(p_{i}\right)$ 's, and $m_{i}-1$ the ramification index at $p \in \pi_{\Delta_{0}}^{-1}\left(r_{i}^{\prime}\right)$. Note $2 \leq m_{i} \leq\left|\Delta_{0}\right|$, and (3-8) can be rewritten as

$$
4 \gamma+2=\sum_{i=1}^{s}\left(m_{i}-1\right) \frac{\left|\Delta_{0}\right|}{m_{i}}=2(\gamma+1) \sum_{i=1}^{s}\left(1-\frac{1}{m_{i}}\right) \text {. }
$$

If $s=1$, then (3-9) yields $2 \gamma<0$, which is absurd. Hence $s \geq 2$, and (3-9) becomes

$$
2 \gamma=2(\gamma+1)\left(\sum_{i=1}^{s}\left(1-\frac{1}{m_{i}}\right)-1\right)=2(\gamma+1)\left(\frac{s-2}{2}+\sum_{i=1}^{s}\left(\frac{1}{2}-\frac{1}{m_{i}}\right)\right) .
$$

So

$$
1>\frac{2 \gamma}{2(\gamma+1)}=\frac{s-2}{2}+\sum_{i=1}^{s}\left(\frac{1}{2}-\frac{1}{m_{i}}\right) \geq \frac{s-2}{2} .
$$

As a result, $2 \leq s<4$ follows, and thus $s=2$ or 3 .

The case $s=2$.

Equation (3-9) implies

$$
2 \gamma=2(\gamma+1)\left(1-\frac{1}{m_{1}}-\frac{1}{m_{2}}\right),
$$

that is,

$$
\frac{1}{m_{1}}+\frac{1}{m_{2}}=\frac{1}{\gamma+1}
$$

holds. The inequalities $2 \leq m_{i} \leq\left|\Delta_{0}\right|=2(\gamma+1)$ yield $m_{1}=m_{2}=2(\gamma+1)$. 
The case $s=3$.

From (3-9),

$$
2 \gamma=2(\gamma+1)\left(2-\frac{1}{m_{1}}-\frac{1}{m_{2}}-\frac{1}{m_{3}}\right) .
$$

Without loss of generality, we may assume $m_{1} \leq m_{2} \leq m_{3}$. In this case,

$$
2-\frac{3}{m_{1}} \leq 2-\frac{1}{m_{1}}-\frac{1}{m_{2}}-\frac{1}{m_{3}} \leq 2-\frac{3}{m_{3}} \text {. }
$$

By (3-10), we obtain

$$
2(\gamma+1)\left(2-\frac{3}{m_{1}}\right) \leq 2(\gamma+1)\left(2-\frac{1}{m_{1}}-\frac{1}{m_{2}}-\frac{1}{m_{3}}\right)=2 \gamma .
$$

Thus we have

$$
m_{1} \leq \frac{3(\gamma+1)}{\gamma+2}<3
$$

Hence $m_{1}=2$. Moreover, let us consider the case $m_{1}=2$ and $m_{2} \leq m_{3}$. Then

$$
2(\gamma+1)\left(\frac{3}{2}-\frac{2}{m_{2}}\right) \leq 2(\gamma+1)\left(2-\frac{1}{2}-\frac{1}{m_{2}}-\frac{1}{m_{3}}\right)=2 \gamma,
$$

and so

$$
m_{2} \leq \frac{4(\gamma+1)}{\gamma+3}<4
$$

It follows that $m_{2}=2$ or 3 . For the case $m_{2}=2$, we have

$$
2 \gamma=2(\gamma+1)\left(1-\frac{1}{m_{3}}\right),
$$

and thus $m_{3}=\gamma+1$. If $m_{2}=3$, then

$$
2 \gamma=2(\gamma+1)\left(\frac{7}{6}-\frac{1}{m_{3}}\right)
$$

and so $m_{3}=\frac{6(\gamma+1)}{\gamma+7}<6$. As a consequence, $\left(m_{3}, \gamma\right)=(3,5),(4,11)$, or $(5,29)$.

Case 2. The point $p_{1}$ cannot be transformed to $p_{2}$.

If there does not exist $\sigma$ satisfying $\sigma\left(p_{1}\right)=p_{2}$, then the ramification index at $p_{i}$ must be $\left|\Delta_{0}\right|-1$. It follows that (3-6) can be reduced to

$$
2 \gamma-2=\left|\Delta_{0}\right|\left(2 \gamma\left(\bar{M} / \Delta_{0}\right)-2\right)+2\left(\left|\Delta_{0}\right|-1\right)+\sum_{p \in M}(\mu(p)-1),
$$

and thus

$$
\begin{aligned}
2 \gamma & =2\left|\Delta_{0}\right| \gamma\left(\bar{M} / \Delta_{0}\right)+\sum_{p \in M}(\mu(p)-1) \\
& =4(\gamma+1) \gamma\left(\bar{M} / \Delta_{0}\right)+\sum_{p \in M}(\mu(p)-1) .
\end{aligned}
$$




\begin{tabular}{c|cccc}
$s$ & \multicolumn{4}{|c}{$m_{i}$} \\
\hline$s=2$ & \multicolumn{3}{|c}{$m_{1}=m_{2}=2(\gamma+1)$} \\
\hline \multirow{4}{*}{$s=3$} & $m_{1}$ & $m_{2}$ & $m_{3}$ & $\gamma$ \\
\cline { 2 - 5 } & 2 & 2 & $\gamma+1$ & arbitrary \\
& 2 & 3 & 3 & 5 \\
2 & 3 & 4 & 11 \\
& 2 & 3 & 5 & 29 \\
\hline
\end{tabular}

Table 6. The case $p_{1}$ can be transformed to $p_{2}$.

\begin{tabular}{c|c}
$s$ & $m_{i}$ \\
\hline$s=1$ & $m_{1}=\gamma+1$ \\
\hline
\end{tabular}

Table 7. The case $p_{1}$ cannot be transformed to $p_{2}$.

Equation (3-11) yields $\gamma\left(\bar{M} / \Delta_{0}\right)=0$, and so

$$
2 \gamma=\sum_{p \in M}(\mu(p)-1) .
$$

Suppose that $r_{1}^{\prime}, \ldots, r_{s}^{\prime}$ are ramified values of $\pi_{\Delta_{0}}$ except for the $\pi_{\Delta_{0}}\left(p_{i}\right)$ 's, and $m_{i}-1$ the ramification index at $p \in \pi_{\Delta_{0}}^{-1}\left(r_{i}^{\prime}\right)$ as above. Now (3-12) can be rewritten as

$$
2 \gamma=\sum_{i=1}^{s}\left(m_{i}-1\right) \frac{\left|\Delta_{0}\right|}{m_{i}}=2(\gamma+1) \sum_{i=1}^{s}\left(1-\frac{1}{m_{i}}\right) .
$$

As a result,

$$
1>\frac{2 \gamma}{2 \gamma+2}=\sum_{i=1}^{s}\left(1-\frac{1}{m_{i}}\right)=\sum_{i=1}^{s}\left(\frac{1}{2}+\left(\frac{1}{2}-\frac{1}{m_{i}}\right)\right) \geq \frac{s}{2},
$$

and hence $1 \leq s<2$, that is, $s=1$. Thus, we have $m_{1}=\gamma+1$.

Therefore, we obtain Tables 6 and 7.

3B. Weierstrass data for the case $\gamma=1$ with $\left(d_{1}, d_{2}\right)=(1,3)$. By Tables $3-5$, we first consider the case $t=2$. Then $|\mathcal{R}|=2$ and we find $\left|\Delta_{0}\right|=4$ by (3-1). Set $q_{1}, q_{2}$ as two branch points of $\pi_{\mathcal{R}}$ distinct from the $p_{i}$ 's and $p_{i}^{\prime}:=\pi_{\mathcal{R}}\left(p_{i}\right), q_{i}^{\prime}:=\pi_{\mathcal{R}}\left(q_{i}\right)$. Since $\pi_{\mathcal{R}}$ is a cyclic branched double cover of $S^{2}, \bar{M}_{1}$ can be given by

$$
v^{2}=\left(u-p_{1}^{\prime}\right)^{m_{1} h_{1}}\left(u-p_{2}^{\prime}\right)^{m_{2} h_{2}}\left(u-q_{1}^{\prime}\right)^{m_{3} h_{3}}\left(u-q_{2}^{\prime}\right)^{m_{4}},
$$

where $h_{i} \in\{1,-1\}(i=1,2,3),\left(2, m_{i}\right)=1$, and $R(u, v)=(u,-v)$.

Since $\left(d_{1}, d_{2}\right)=(1,3)$, there does not exist $\sigma \in \Delta_{0}$ with $\sigma\left(p_{1}\right)=p_{2}$. By Table 7 on $\Delta_{0}$, there is a transformation $\tau \in \Delta_{0} \backslash \mathcal{R}$ satisfying $\tau\left(q_{1}\right)=q_{2}$, and thus $m_{3}=m_{4}$. 


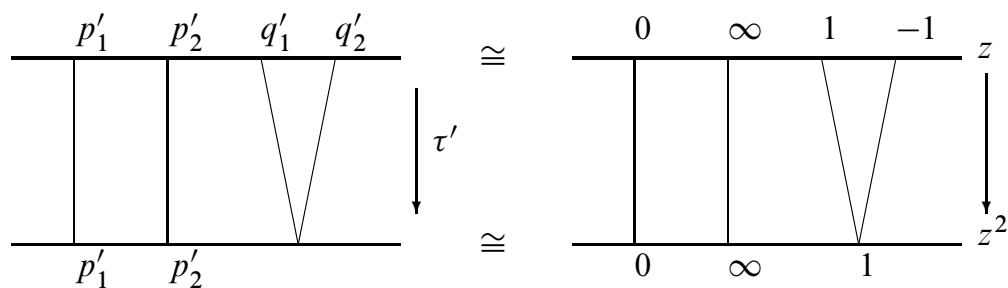

Figure 9. The Riemann surface $\bar{M}_{1}$.
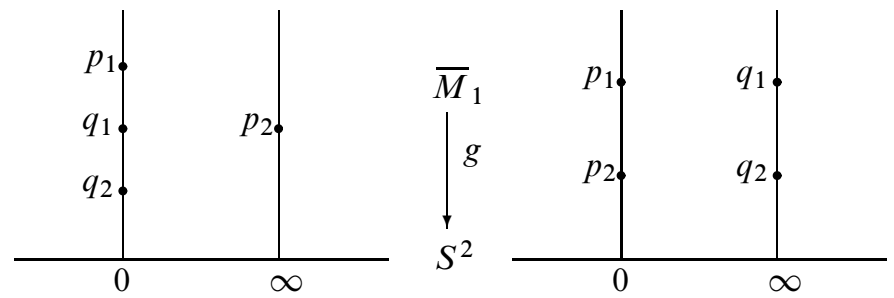

Figure 10. The possibilities of the Gauss map.

The transformation $\tau$ induces a degree 2 transformation $\tau^{\prime}: \bar{M}_{1} / \mathcal{R} \rightarrow \bar{M}_{1} / \Delta_{0}$, that is, a transformation on $S^{2}$ such that $\tau^{\prime}\left(q_{1}^{\prime}\right)=\tau^{\prime}\left(q_{2}^{\prime}\right)$. Choosing suitable variables $(z, w)$, we can represent $\tau(z, w)=(-z, *), \tau^{\prime}(z)=z^{2}, p_{1}^{\prime}=0, p_{2}^{\prime}=\infty, q_{1}^{\prime}=1, q_{2}^{\prime}=-1$, and moreover, $\bar{M}_{1}$ can be rewritten as $w^{2}=z\left(z^{2}-1\right)$ (see Figure 9).

Now consider the Gauss map. Since $p_{1}, p_{2}, q_{1}, q_{2}$ are fixed points of $\mathcal{R}$ (that is, fixed points by rotations around the $x_{3}$-axis), we have $g\left(\left\{p_{1}, p_{2}, q_{1}, q_{2}\right\}\right) \subset\{0, \infty\}$. Note that $q_{1}$ can be transformed to $q_{2}$ by the biholomorphism $\tau$. On the other hand, $p_{1}$ cannot be transformed to $p_{2}$. It follows that we essentially only need to consider the two cases depicted in Figure 10.

In the right-hand side case in Figure 10, the ramification index at $q_{i}$ is $\frac{\gamma+2}{2}-1 \notin \mathbb{Z}$ since the ramification index at $q_{1}$ of $g$ must coincide with the ramification index at $q_{2}$ of $g$. Hence we only consider the left-hand side case in Figure 10. The ramification index at $p_{2}$ may be $1-1,2-1$, or $3-1$. If it is $2-1$, then $g^{-1}(\infty)$ consists of $p_{2}$ and a simple pole $q \in \bar{M}_{1}$. Then $R(q)$ must be a pole of $g$, but $R(q) \notin\left\{p_{2}, q\right\}$. This contradicts $R(q) \in g^{-1}(\infty)$. So the divisor of $g$ is given by

$$
(g)=\left\{\begin{array}{l}
p_{1}+q_{1}+q_{2}-3 p_{2}, \\
p_{1}+q_{1}+q_{2}-p_{2}-Q-R(Q)
\end{array}\right.
$$

for a point $Q$. Since $\tau\left(p_{2}\right)=p_{2}, \tau$ leaves \{the poles of $g$ \} invariant. For the latter case, if we take $Q^{*}:=\tau(Q)$, then $Q^{*}$ must be a pole of $g$ which is distinct from the $R^{i}(Q)$ 's. It leads to a contradiction, and so we only consider the former case. In this case, the divisor of the meromorphic function $z\left(z^{2}-1\right)$ coincides with that 
of $g^{2}$, thus $g^{2}=c^{\prime} z\left(z^{2}-1\right)$ holds for some constant $c^{\prime}$. Hence, $\bar{M}_{1}$ and $g$ can be rewritten as

$$
w^{2}=z\left(z^{2}-1\right), \quad g=c w
$$

for some constant $c$, and $R(z, w)=(z,-w), \tau(z, w)=(-z, i w)$. Then, the divisor of $\eta$ is obtained by

$$
(\eta)=\left\{\begin{array}{l}
-2 p_{1}+2 p_{2} \\
-4 p_{1}+4 p_{2}
\end{array}\right.
$$

since $\left(d_{1}, d_{2}\right)=(1,3)$. Thus, by a similar argument,

$$
\eta^{2}=\left\{\begin{array}{l}
c^{\prime \prime} \frac{(d z)^{2}}{z^{3}\left(z^{2}-1\right)} \\
c^{\prime \prime} \frac{(d z)^{2}}{z^{5}\left(z^{2}-1\right)}
\end{array}=\left\{\begin{array}{l}
c^{\prime \prime}\left(\frac{d z}{z w}\right)^{2} \\
c^{\prime \prime}\left(\frac{d z}{z^{2} w}\right)^{2}
\end{array}\right.\right.
$$

hold for some constant $c^{\prime \prime}$. As a consequence, we obtain $\eta=c^{\prime \prime \prime} \frac{d z}{z w}, c^{\prime \prime \prime} \frac{d z}{z^{2} w}$ for some constant $c^{\prime \prime \prime}$. The latter case is given in Section 2A, and we shall prove that the former case does not occur in Section 3D. Note that the case $t=1$ does not occur in this case.

3C. Weierstrass data for the even genus case with $\left(d_{1}, d_{2}\right)=(2,2)$. We treat the case $t=2\left(|\mathcal{R}|=\gamma+1,\left|\Delta_{0}\right|=2(\gamma+1)\right)$. By Table $4, \pi_{\mathcal{R}}: \bar{M}_{\gamma} \rightarrow \bar{M}_{\gamma} / \mathcal{R}$ is a cyclic branched cover of $S^{2}$. Thus $\bar{M}_{\gamma}$ can be represented by

$$
v^{\gamma+1}=\left(u-p_{1}^{\prime}\right)^{m_{1} h_{1}}\left(u-p_{2}^{\prime}\right)^{m_{2} h_{2}}\left(u-q_{1}^{\prime}\right)^{m_{3} h_{3}}\left(u-q_{2}^{\prime}\right)^{m_{4}},
$$

where $\left(m_{i}, \gamma+1\right)=1, h_{i}= \pm 1$, and $R(u, v)=\left(u, e^{2 \pi i /(\gamma+1)} v\right)$.

Case 1. The point $p_{1}$ cannot be transformed to $p_{2}$.

We assume that there does not exist $\sigma \in \Delta_{0}$ such that $\sigma\left(p_{1}\right)=p_{2}$. From the table on $\Delta_{0}$, there exists a transformation $\tau \in \Delta_{0} \backslash \mathcal{R}$ satisfying $\tau\left(q_{1}\right)=q_{2}$, and thus $m_{3}=m_{4}$. This $\tau$ induces a degree 2 transformation $\tau^{\prime}: \bar{M}_{1} / \mathcal{R} \rightarrow \bar{M}_{1} / \Delta_{0}$, that is, a transformation on $S^{2}$ such that $\tau^{\prime}\left(q_{1}^{\prime}\right)=\tau^{\prime}\left(q_{2}^{\prime}\right)$. Choosing suitable variables $(z, w)$, we can represent $\tau(z, w)=(-z, *), \tau^{\prime}(z)=z^{2}, p_{1}^{\prime}=0, p_{2}^{\prime}=\infty, q_{1}^{\prime}=1$, $q_{2}^{\prime}=-1$, and moreover, $\bar{M}_{\gamma}$ can be rewritten as $w^{\gamma+1}=z^{m_{1} h_{1}}\left(z^{2}-1\right)^{m_{3}}$ and $R(z, w)=\left(z, e^{2 \pi i /(\gamma+1)} w\right)$ (see Figure 11).

Now we consider the Gauss map. Since $p_{1}, p_{2}, q_{1}, q_{2}$ are fixed points of $\mathcal{R}$ (that is, fixed points by rotations around the $x_{3}$-axis), we find $g\left(\left\{p_{1}, p_{2}, q_{1}, q_{2}\right\}\right) \subset\{0, \infty\}$. Note that $q_{1}$ can be transformed to $q_{2}$ by the biholomorphism $\tau$. On the other hand, $p_{1}$ cannot be transformed to $p_{2}$. It follows that we essentially only need to consider the two cases as in Figure 12.

The left-hand side case in Figure 12. 


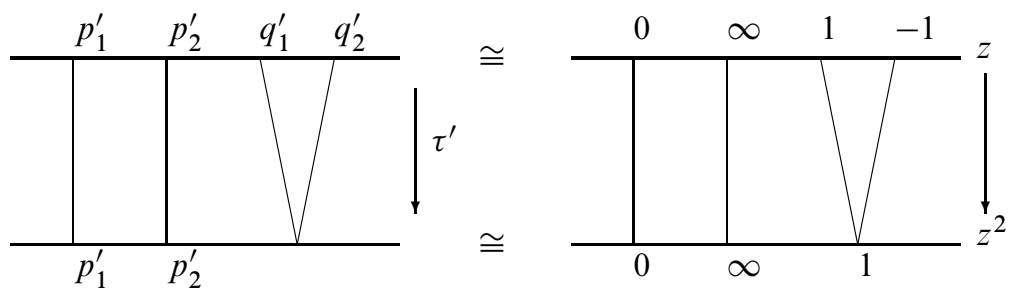

Figure 11. The Riemann surface $\bar{M}_{\gamma}$.
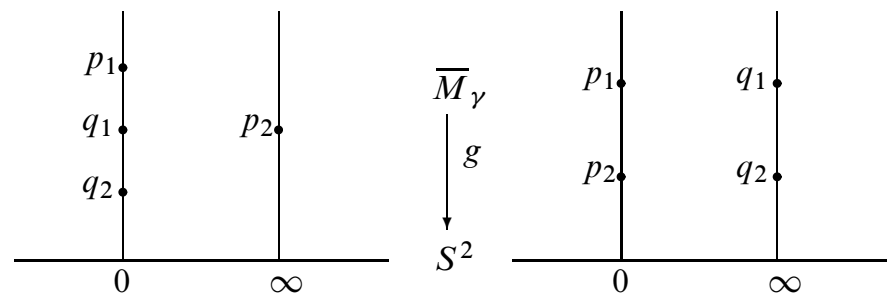

Figure 12. The possibilities of the Gauss map.

The divisor of $g$ is given by

$$
(g)=\left\{\begin{array}{l}
(\gamma+2-2 N) p_{1}+N q_{1}+N q_{2}-(\gamma+2) p_{2} \\
(\gamma+2-2 N) p_{1}+N q_{1}+N q_{2}-p_{2}-Q-R(Q)-\cdots-R^{\gamma}(Q)
\end{array}\right.
$$

for a point $Q$. Note that $N>0$ and $\gamma+2-2 N>0$. Since $\tau\left(p_{2}\right)=p_{2}, \tau$ leaves \{the poles of $g$ \} invariant. For the latter case, if we take $Q^{*}:=\tau(Q)$, then $Q^{*}$ must be a pole of $g$ which is distinct from the $R^{i}(Q)$ 's. This leads to a contradiction, and so we only consider the former case. Then the divisor of $\eta$ is given by

$$
(\eta)=-3 p_{1}+(2 \gamma+1) p_{2}
$$

Hence the divisor of $g \eta$ is obtained by

$$
(g \eta)=(\gamma-2 N-1) p_{1}+N q_{1}+N q_{2}+(\gamma-1) p_{2} .
$$

If $\gamma-2 N-1 \geq 0$, then $g \eta$ is holomorphic. Thus $f$ is bounded and this leads to a contradiction. As a result, $\gamma-2 N-1<0$ follows. The inequality $\gamma+2-2 N>0$ yields $N<\frac{\gamma}{2}+1$. Also, since $\gamma$ is even, $N \leq \frac{\gamma}{2}$ holds. So we have $\gamma=2 N$.

It follows that the divisor of $g^{\gamma+1}$ coincides with that of $z^{2}\left(z^{2}-1\right)^{\gamma / 2}$. Therefore, $\bar{M}_{\gamma}$ and $g$ can be rewritten as

$$
w^{\gamma+1}=z^{2}\left(z^{2}-1\right)^{\gamma / 2}, \quad g=c w
$$


for some constant $c$, and $R(z, w)=\left(z, e^{2 \pi i /(\gamma+1)} w\right), \tau(z, w)=(-z, w)$. Furthermore, the divisor of $\eta^{\gamma+1}$ coincides with that of $(d z)^{\gamma+1} /\left(z^{\gamma-1} g^{2(\gamma+1)}\right)$. Hence

$$
\eta^{\gamma+1}=c^{\prime \prime} \frac{(d z)^{\gamma+1}}{z^{\gamma-1} g^{2(\gamma+1)}}\left(=c^{\prime \prime} z^{2} \frac{(d z)^{\gamma+1}}{z^{\gamma+1} g^{2(\gamma+1)}}\right)
$$

for some constant $c^{\prime \prime}$. By setting $z=u^{\gamma+1}$ and $w=u^{2} v, \bar{M}_{\gamma}$ can be rewritten as

$$
v^{\gamma+1}=\left(u^{2(\gamma+1)}-1\right)^{\gamma / 2},
$$

and moreover,

$$
g=c u^{2} v, \quad \eta=c^{\prime \prime \prime} \frac{u}{g^{2}} d u
$$

for some constant $c^{\prime \prime \prime}$. However, in this case, its genus is greater than $\gamma$, and such a case is excluded.

The right-hand side case in Figure 12.

The divisor of $g$ is obtained by

$$
(g)=(\gamma+2-N) p_{1}+N p_{2}-\frac{\gamma+2}{2} q_{1}-\frac{\gamma+2}{2} q_{2},
$$

where $N>0$ and $\gamma+2-N>0$. Also, the divisor of $\eta$ is given by

$$
(\eta)=-3 p_{1}-3 p_{2}+(\gamma+2) q_{1}+(\gamma+2) q_{2} .
$$

Thus the divisor of $g \eta$ is obtained by

$$
(g \eta)=(\gamma-N-1) p_{1}+(N-3) p_{2}+\frac{\gamma+2}{2} q_{1}+\frac{\gamma+2}{2} q_{2} .
$$

If $\gamma-N-1 \geq 0$ and $N-3 \geq 0$, then $g \eta$ is holomorphic. Hence $\gamma-N-1<0$ or $N-3<0$. From the inequality $\gamma+2-N>0$, the case $\gamma-N-1<0$ corresponds to $\gamma=N, N-1$. The case $N-3<0$ implies $N=1$ or 2 . Essentially, we consider the cases $N=1,2$. The divisor of $g^{\gamma+1}$ coincides with

$$
\frac{z^{\gamma+2-N}}{\left(z^{2}-1\right)^{(\gamma+2) / 2}} \text {. }
$$

As a consequence, $\bar{M}_{\gamma}$ and $g$ can be rewritten as

$$
w^{\gamma+1}=\frac{\left(z^{2}-1\right)^{(\gamma+2) / 2}}{z^{\gamma+2-N}}, \quad g=\frac{c}{w}
$$

for some constant $c$. If $N=1$, then $\gamma+2-N$ and $\gamma+1$ are not coprime. So $N=2$, and $R(z, w)=\left(z, e^{2 \pi i /(\gamma+1)} w\right), \tau(z, w)=(-z, w)$. Furthermore, the divisor $\eta^{\gamma+1}$ coincides with that of

$$
\frac{\left(z^{2}-1\right)^{2}}{z^{\gamma+3}}(d z)^{\gamma+1}
$$




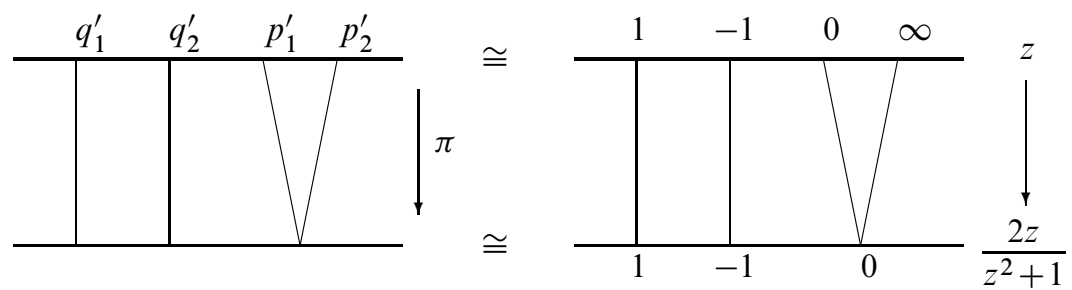

Figure 13. The Riemann surface $\bar{M}_{\gamma}$ (for the case $s=2$ ).

As a consequence,

$$
\eta^{\gamma+1}=c^{\prime} \frac{\left(z^{2}-1\right)^{2}}{z^{\gamma+3}}(d z)^{\gamma+1}\left(=\frac{c^{\prime}}{z^{6}}\left(\frac{z^{3} w^{4} d z}{\left(z^{2}-1\right)^{2}}\right)^{\gamma+1}\right)
$$

for some constant $c^{\prime}$. By similar arguments as above, we may exclude this case except the case $\gamma=2$. For the case $\gamma=2, \bar{M}_{\gamma}$ and $g$ can be rewritten as

$$
w^{3}=\frac{\left(z^{2}-1\right)^{2}}{z^{2}}, \quad g=\frac{c}{w},
$$

and moreover, we find $\eta=c^{\prime \prime} \frac{w}{z} d z$ for some constants $c, c^{\prime \prime}$. However, this surface has a transformation $\sigma \in \Delta_{0}$ defined by $\sigma(z, w)=\left(\frac{1}{z}, w\right)$, and we have $\sigma\left(p_{1}\right)=p_{2}$. This contradicts our assumption.

Case 2. The point $p_{1}$ can be transformed to $p_{2}$.

Suppose that there exists $\sigma \in \Delta_{0}$ such that $\sigma\left(p_{1}\right)=p_{2}$. By Table 6, we consider two cases, namely the case $s=2$ and the case $s=3$. Note that $\sigma\left(p_{2}\right)=p_{1}$ and $\sigma \in \Delta_{0} \backslash \mathcal{R}$.

The case $s=2$.

By Table 6 , every $q_{i}$ must be branch points of $\pi_{\Delta_{0}}$ with the ramified index $2(\gamma+1)-1$. Hence, $\sigma\left(q_{i}\right)=q_{i}$ for $i=1,2$, and moreover, $\sigma$ induces a degree 2 transformation $\sigma^{\prime}: \bar{M}_{\gamma} / \mathcal{R} \rightarrow \bar{M}_{\gamma} / \Delta_{0}$ which is a transformation on $S^{2}$ and the $q_{i}^{\prime}$ ss are two fixed points of $\sigma^{\prime}$ (see Figure 13).

By suitable variables $(z, w)$, we have $\sigma(z, w)=\left(\frac{1}{z}, *\right), \sigma^{\prime}(z)=\frac{2 z}{z^{2}+1}, p_{1}^{\prime}=0$, $p_{2}^{\prime}=\infty, q_{1}^{\prime}=1, q_{2}^{\prime}=-1$. Also, $\bar{M}_{\gamma}$ is given by

$$
w^{2}=z^{m_{1} h_{1}}(z-1)^{m_{2} h_{2}}(z+1)^{m_{3} h_{3}} \text {. }
$$

We consider the Gauss map. Essentially, we treat the two cases shown in Figure 14.

For the case in the left-hand side of Figure 14, the divisor of $g$ is given by

$$
(g)=\left\{\begin{array}{l}
(\gamma+2-2 N) q_{1}+N p_{1}+N p_{2}-(\gamma+2) q_{2} \\
(\gamma+2-2 N) q_{1}+N p_{1}+N p_{2}-q_{2}-Q-R(Q)-\cdots-R^{\gamma}(Q),
\end{array}\right.
$$



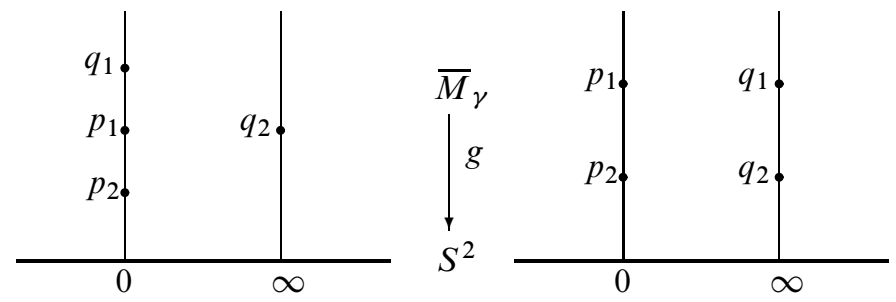

Figure 14. The possibilities of the Gauss map.

where $N>0$ and $\gamma+2-2 N>0$. Since $\sigma\left(q_{2}\right)=q_{2}, \sigma$ leaves \{the poles of $g$ \} invariant. For the latter case, if we take $Q^{*}:=\sigma(Q)$, then $Q^{*}$ must be a pole of $g$ which is distinct from the $Q_{i}$ 's. This leads to a contradiction, and so we only consider the former case. In this case, we see

$$
(\eta)=-3 p_{1}-3 p_{2}+(2 \gamma+4) q_{2} .
$$

Then

$$
(g \eta)=(\gamma+2-2 N) q_{1}+(N-3) p_{1}+(N-3) p_{2}+(\gamma+2) q_{2} .
$$

It follows that $N-3<0$. With $N>0$, this yields $N=1$ or 2 .

Thus the divisor of $g^{\gamma+1}$ coincides with that of

$$
\frac{z^{N}(z-1)^{\gamma+2-2 N}}{(z+1)^{\gamma+2}}
$$

Therefore, $\bar{M}_{\gamma}$ and $g$ can be rewritten as

$$
w^{\gamma+1}=\frac{z^{N}(z-1)^{\gamma+2-2 N}}{(z+1)^{\gamma+2}} \quad(\text { for } N=1,2), \quad g=c w
$$

for some constant $c$, and $R(z, w)=\left(z, e^{2 \pi i /(\gamma+1)} w\right), \sigma(z, w)=\left(\frac{1}{z}, w\right)$. Furthermore, by similar arguments, $\eta$ can be obtained by

$$
\eta^{\gamma+1}=c^{\prime} \frac{(z+1)^{\gamma+4}}{z^{\gamma+3}(z-1)^{\gamma}}(d z)^{\gamma+1}= \begin{cases}c^{\prime} \frac{z+1}{z-1}\left(\frac{(z-1) d z}{z(z+1) w^{2}}\right)^{\gamma+1} & \text { for } N=1, \\ c^{\prime}\left(\frac{z+1}{z-1}\right)^{2}\left(\frac{d z}{z w}\right)^{\gamma+1} & \text { for } N=2,\end{cases}
$$

for some constant $c^{\prime}$. So we may exclude this case, like the previous case.

Next we consider the case in the right-hand side of Figure 14. Then the divisor of $g$ is obtained by

$$
(g)=\frac{\gamma+2}{2} p_{1}+\frac{\gamma+2}{2} p_{2}-N q_{1}-(\gamma+2-N) q_{2},
$$

where $N>0$ and $\gamma+2-N>0$. In this case, the divisor of $\eta$ is given by

$$
(\eta)=-3 p_{1}-3 p_{2}+2 N q_{1}+(2 \gamma+4-2 N) q_{2} .
$$




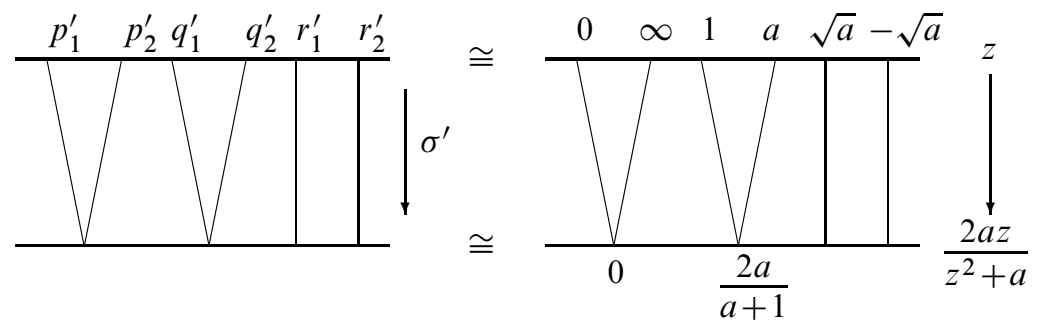

Figure 15. The Riemann surface $\bar{M}_{\gamma}$ (the case $s=3$ ).

Thus

$$
(g \eta)=\frac{\gamma-4}{2} p_{1}+\frac{\gamma-4}{2} p_{2}+N q_{1}+(\gamma+2-N) q_{2} .
$$

Hence $\gamma-4<0$ and so $\gamma=2$. Moreover, from the inequality $\gamma+2-N>0$, we obtain $N=1,2$, or 3 . If $N=1$, then $\gamma+2-N$ and $\gamma+1$ are not coprime. Also, if $N=3$, then $N$ and $\gamma+1$ are not coprime. So we have $N=2$. As a result, the divisor of $g^{\gamma+1}\left(=g^{3}\right)$ coincides with that of

$$
\frac{z^{(\gamma+2) / 2}}{(z-1)^{N}(z+1)^{\gamma+2-N}}\left(=\frac{z^{2}}{(z-1)^{2}(z+1)^{2}}\right) .
$$

Therefore, $\bar{M}_{2}$ and $g$ can be rewritten as

$$
w^{3}=\frac{(z-1)^{2}(z+1)^{2}}{z^{2}}, \quad g=\frac{c}{w}
$$

for some constant $c$, and $R(z, w)=\left(z, e^{2 \pi i / 3} w\right), \sigma(z, w)=\left(\frac{1}{z}, w\right)$. Also, we have $\eta=c^{\prime} \frac{w}{z} d z$ for some constant $c^{\prime}$. We shall prove that this case does not occur in Section 3D.

The case $s=3$

From Table 6, we have that the $p_{i}$ 's and $q_{i}$ 's must be branch points of $\pi_{\Delta_{0}}$ with the ramified index $(\gamma+1)-1$. As a result, there exist two sets $\left\{r_{1}^{(1)}, \ldots, r_{\gamma+1}^{(1)}\right\}$, $\left\{r_{1}^{(2)}, \ldots, r_{\gamma+1}^{(2)}\right\}$ of branch points with the ramified index $2-1$ of $\pi_{\Delta_{0}}$ satisfying $\pi_{\Delta_{0}}\left(r_{i}^{(1)}\right)=\pi_{\Delta_{0}}\left(r_{j}^{(1)}\right)$ and $\pi_{\Delta_{0}}\left(r_{i}^{(2)}\right)=\pi_{\Delta_{0}}\left(r_{j}^{(2)}\right)$ for $1 \leq i, j \leq \gamma+1$. Note that $r_{i}^{(1)}$ and $r_{i}^{(2)}$ are distinct from the $p_{i}$ 's and $q_{i}$ 's. Hence, $\sigma\left(q_{1}\right)=q_{2}$ and $\sigma\left(q_{2}\right)=q_{1}$, and moreover, $\sigma$ induces a degree 2 transformation $\sigma^{\prime}: \bar{M}_{\gamma} / \mathcal{R} \rightarrow \bar{M}_{\gamma} / \Delta_{0}$, that is, a transformation on $S^{2}$ such that $r_{i}^{\prime}:=\pi_{\mathcal{R}}\left(r_{j}^{(i)}\right)$ 's are two branch points of $\sigma^{\prime}$ (see Figure 15).

Choosing suitable variables $(z, w)$, for $a \in \mathbb{C} \backslash\{0\}$, we have $\sigma(z, w)=\left(\frac{a}{z}, *\right)$, $\sigma^{\prime}(z)=2 a z /\left(z^{2}+a\right), p_{1}^{\prime}=0, p_{2}^{\prime}=\infty, q_{1}^{\prime}=1, q_{2}^{\prime}=a, r_{1}^{\prime}=\sqrt{a}, r_{2}^{\prime}=-\sqrt{a}$. Also, $\bar{M}_{\gamma}$ is given by $w^{\gamma+1}=z^{m_{1} h_{1}}(z-1)^{m_{2} h_{2}}(z-a)^{m_{2} h_{3}}$ (see Figure 15).

We now consider the Gauss map, essentially the two cases in Figure 16. 

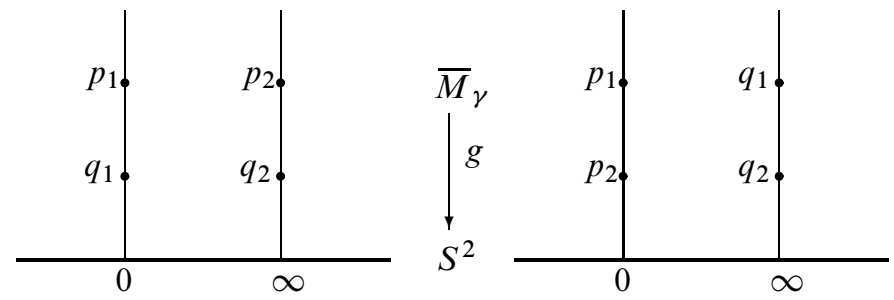

Figure 16. The possibilities of the Gauss map.

Then the divisor of $g$ is obtained by

$$
(g)= \begin{cases}(\gamma+2-N) p_{1}+N q_{1}-(\gamma+2-N) p_{2}-N q_{2} & (\text { the LHS case), } \\ \frac{\gamma+2}{2} p_{1}+\frac{\gamma+2}{2} p_{2}-\frac{\gamma+2}{2} q_{1}-\frac{\gamma+2}{2} q_{2} & \text { (the RHS case), }\end{cases}
$$

where $N>0$ and $\gamma+2-N>0$. The divisor of $\eta$ is given by

$$
(\eta)=\left\{\begin{array}{l}
-3 p_{1}+(2 \gamma-2 N+1) p_{2}+2 N q_{2} \\
-3 p_{1}-3 p_{2}+(\gamma+2) q_{1}+(\gamma+2) q_{2}
\end{array}\right.
$$

So the divisor of $g \eta$ is obtained by

$$
(g \eta)=\left\{\begin{array}{l}
(\gamma-N-1) p_{1}+(\gamma-N-1) p_{2}+N q_{1}+N q_{2}, \\
\frac{\gamma-4}{2} p_{1}+\frac{\gamma-4}{2} p_{2}+\frac{\gamma+2}{2} q_{1}+\frac{\gamma+2}{2} q_{2} .
\end{array}\right.
$$

For the former case, if $\gamma-N-1 \geq 0$, then $g \eta$ is holomorphic. Thus $\gamma-N-1<0$ holds. Also, the inequality $\gamma+2-N>0$ yields $N=\gamma$ or $\gamma+1$. For the latter case, $\gamma-4 \geq 0$ cannot hold, and hence $\gamma=2$. As a consequence,

$$
g^{\gamma+1}= \begin{cases}c^{\prime} z^{\gamma+2-N}\left(\frac{z-1}{z-a}\right)^{N} & (\text { the LHS case) }, \\ c^{\prime}\left(\frac{z}{(z-1)(z-a)}\right)^{(\gamma+2) / 2} & \text { (the RHS case) },\end{cases}
$$

for some constant $c^{\prime}$. If $N=\gamma+1$, then $\gamma+1$ and $N$ are not coprime. Thus $N=\gamma$ follows. Therefore, $\bar{M}_{\gamma}$ and $g$ can be rewritten as

$$
\left\{\begin{array}{l}
w^{\gamma+1}=z^{2}\left(\frac{z-1}{z-a}\right)^{\gamma}, \quad g=c w \quad \text { (the LHS case), } \\
w^{3}=\left(\frac{(z-1)(z-a)}{z}\right)^{2}, \quad g=\frac{c}{w} \quad \text { (the RHS case), }
\end{array}\right.
$$


and

$$
\begin{aligned}
& R(z, w)=\left(z, e^{2 \pi i /(\gamma+1)} w\right), \\
& \sigma(z, w)= \begin{cases}\left(\frac{a}{z}, \frac{a^{(\gamma+2) /(\gamma+1)}}{w}\right) & \text { (the LHS case), } \\
\left(\frac{a}{z}, w\right) & \text { (the RHS case) } .\end{cases}
\end{aligned}
$$

Also, for some constant $c^{\prime}$, we have

$$
\eta= \begin{cases}c^{\prime} \frac{d z}{z w} & \text { (the LHS case) } \\ c^{\prime} \frac{w}{z} d z & \text { (the RHS case). }\end{cases}
$$

$\Delta \backslash \Delta_{0} \neq \varnothing$ implies that there exists a degree 2 antiholomorphic transformation. Hence $a \in \mathbb{R}$ and the antiholomorphic transformation can be represented by the map $(z, w) \mapsto(\bar{z}, \bar{w})$. The former case corresponds to our result in Section $2 \mathrm{~B}$, and the latter case is considered in Section 3D. Note that the case $t=1$ does not occur.

3D. Well-definedness. In this subsection, we consider the well-definedness for the following two cases:

\begin{tabular}{c|c|c|c}
$\bar{M}_{\gamma}$ & $g$ & $\eta$ & symmetries \\
\hline$w^{2}=z\left(z^{2}-1\right)$ & $c w$ & $c^{\prime} \frac{d z}{z w}$ & $R(z, w)=(-z, i w)$ \\
\hline$w^{3}=\frac{(z-1)^{2}(z-a)^{2}}{z^{2}}$ & $\frac{c}{w}$ & $c^{\prime} \frac{w}{z} d z$ & $\begin{array}{l}R(z, w)=\left(z, e^{2 \pi i / 3} w\right), \\
\sigma(z, w)=\left(\frac{a}{z}, w\right)\end{array}$ \\
\hline
\end{tabular}

Note that $c, c^{\prime} \in \mathbb{C} \backslash\{0\}, a \in \mathbb{R} \backslash\{0,1\}$. $M$ is given by

$$
M= \begin{cases}\bar{M}_{1} \backslash\{(0,0),(\infty, \infty)\} & \text { (the former case) } \\ \bar{M}_{2} \backslash\{(0, \infty),(\infty, \infty)\} & \text { (the latter case) }\end{cases}
$$

The case $a=-1$ corresponds to the surface which we treat for the case $s=2$. Note that the Weierstrass data $\left(e^{i \theta} g, e^{-i \theta} \eta\right)$ produces the same minimal surface as $(g, \eta)$ rotated by an angle $\theta$ around the $x_{3}$-axis. So after a suitable rotation of the surface, we may assume $c \in \mathbb{R}_{+}$. Also, multiplying a positive real number into $\eta$ is just a homothety, so we may assume that $\left|c^{\prime}\right|=1$.

Our claim is that all cases do not occur.

The former case.

First we consider $\Phi={ }^{t}\left(\Phi_{1}, \Phi_{2}, \Phi_{3}\right)$ in Theorem 1.1:

$$
\Phi_{1}=\left(\frac{1}{w}-c^{2} w\right) c^{\prime} \frac{d z}{z}, \quad \Phi_{2}=i\left(\frac{1}{w}+c^{2} w\right) c^{\prime} \frac{d z}{z}, \quad \Phi_{3}=2 c c^{\prime} \frac{d z}{z} .
$$


For the residue of $\Phi_{3}$ at $z=0$ to be real, we see that $c^{\prime}= \pm 1$. We may choose $c^{\prime}=1$. We shall use the notation in the proof of Theorem 2.2 for $\gamma=1$.

Straightforward calculation yields

$$
\frac{d z}{z w}-d\left(\frac{2 w}{z}\right)=-\frac{z}{w} d z
$$

Thus we have

$$
\begin{gathered}
\oint_{\ell^{\prime}} \eta=-\oint_{\ell^{\prime}} \frac{z}{w} d z=-\oint_{\ell} \frac{z}{w} d z=-2 i \int_{0}^{1} \sqrt{\frac{t}{1-t^{2}}} d t, \\
\oint_{\ell^{\prime}} g^{2} \eta=c^{2} \oint_{\ell} \frac{w}{z} d z=-2 i c^{2} \int_{0}^{1} \sqrt{\frac{1-t^{2}}{t}} d t .
\end{gathered}
$$

Equation (1-4) implies

$$
-\int_{0}^{1} \sqrt{\frac{t}{1-t^{2}}} d t=c^{2} \int_{0}^{1} \sqrt{\frac{1-t^{2}}{t}} d t
$$

So we have $c^{2}<0$ and this contradicts $c>0$.

The latter case.

First we consider $\Phi={ }^{t}\left(\Phi_{1}, \Phi_{2}, \Phi_{3}\right)$ in Theorem 1.1:

$$
\Phi_{1}=\left(w-\frac{c^{2}}{w}\right) c^{\prime} \frac{d z}{z}, \quad \Phi_{2}=i\left(w+\frac{c^{2}}{w}\right) c^{\prime} \frac{d z}{z}, \quad \Phi_{3}=2 c c^{\prime} \frac{d z}{z} .
$$

For the residue of $\Phi_{3}$ at $z=0$ to be real, we see that $c^{\prime}= \pm 1$. We may choose $c^{\prime}=1$.

We shall show that for any $c>0$ and $a \in \mathbb{R} \backslash\{0,1\}$, the period condition (P) cannot be satisfied.

A straightforward calculation yields

$$
\eta+\frac{3}{2} d w=\left(\frac{w}{z-1}+\frac{w}{z-a}\right) d z
$$

Note that the right-hand side of this equation is a holomorphic differential on $\bar{M}_{2} \backslash\{(\infty, \infty)\}$.

We now consider the following three cases: $a>1,0<a<1, a<0$.

(i) The case $a>1$ :

We set

$$
\begin{aligned}
\ell=\left\{(z, w)=\left(t, \sqrt[3]{\frac{(1-t)^{2}(a-t)^{2}}{t^{2}}}\right) \mid 0 \leq t \leq 1\right\} \\
\cup\left\{(z, w)=\left(-t, e^{2 \pi i / 3} \sqrt[3]{\frac{(1+t)^{2}(a+t)^{2}}{t^{2}}}\right) \mid-1 \leq t \leq 0\right\} .
\end{aligned}
$$


From (3-13), we have

$$
\begin{gathered}
\oint_{\ell} \eta=-\left(1-e^{2 \pi i / 3}\right) \int_{0}^{1}\left(\sqrt[3]{\frac{(a-t)^{2}}{t^{2}(1-t)}}+\sqrt[3]{\frac{(1-t)^{2}}{t^{2}(a-t)}}\right) d t \\
\int_{\ell} g^{2} \eta=c^{2}\left(1-e^{-2 \pi i / 3}\right) \int_{0}^{1} \frac{d t}{\sqrt[3]{t(1-t)^{2}(a-t)^{2}}} .
\end{gathered}
$$

Thus (1-4) is equivalent to

$$
-\int_{0}^{1}\left(\sqrt[3]{\frac{(a-t)^{2}}{t^{2}(1-t)}}+\sqrt[3]{\frac{(1-t)^{2}}{t^{2}(a-t)}}\right) d t=c^{2} \int_{0}^{1} \frac{d t}{\sqrt[3]{t(1-t)^{2}(a-t)^{2}}}
$$

But this is impossible because the left-hand side is a negative real number and the right-hand side is a positive real number.

(ii) The case $0<a<1$ :

We set

$$
\begin{aligned}
\ell=\{(z, w)=(a t, & \left.\left.\sqrt[3]{\frac{(a t-1)^{2}(1-t)^{2}}{t^{2}}}\right) \mid 0 \leq t \leq 1\right\} \\
& \cup\left\{(z, w)=\left(-a t, e^{2 \pi i / 3} \sqrt[3]{\frac{(a t+1)^{2}(1+t)^{2}}{t^{2}}}\right) \mid-1 \leq t \leq 0\right\} .
\end{aligned}
$$

From (3-13), we have

$$
\begin{gathered}
\oint_{\ell} \eta=-\left(1-e^{2 \pi i / 3}\right) \int_{0}^{1}\left(a \sqrt[3]{\frac{(1-t)^{2}}{t^{2}(1-a t)}}+\sqrt[3]{\frac{(1-a t)^{2}}{t^{2}(1-t)}}\right) d t \\
\int_{\ell} g^{2} \eta=c^{2}\left(1-e^{-2 \pi i / 3}\right) \int_{0}^{1} \frac{d t}{\sqrt[3]{t(1-t)^{2}(1-a t)^{2}}} .
\end{gathered}
$$

Thus (1-4) is equivalent to

$$
-\int_{0}^{1}\left(a \sqrt[3]{\frac{(1-t)^{2}}{t^{2}(1-a t)}}+\sqrt[3]{\frac{(1-a t)^{2}}{t^{2}(1-t)}}\right) d t=c^{2} \int_{0}^{1} \frac{d t}{\sqrt[3]{t(1-t)^{2}(1-a t)^{2}}}
$$

but again this is impossible by the same reason as in the case (i).

(iii) The case $a<0$ : 
We set

$$
\begin{array}{r}
\ell=\left\{(z, w)=\left(a t, \sqrt[3]{\frac{(1-a t)^{2}(1-t)^{2}}{t^{2}}}\right) \mid 0 \leq t \leq 1\right\} \\
\cup\left\{(z, w)=\left(-a t, e^{2 \pi i / 3} \sqrt[3]{\frac{(1+a t)^{2}(1+t)^{2}}{t^{2}}}\right) \mid-1 \leq t \leq 0\right\}, \\
\ell^{\prime}=\left\{(z, w)=\left(t, \sqrt[3]{\frac{(1-t)^{2}(t-a)^{2}}{t^{2}}}\right) \mid 0 \leq t \leq 1\right\} \\
\cup\left\{(z, w)=\left(-t, e^{2 \pi i / 3} \sqrt[3]{\frac{(1+t)^{2}(t+a)^{2}}{t^{2}}}\right) \mid-1 \leq t \leq 0\right\} .
\end{array}
$$

From (3-13), we have

$$
\begin{gathered}
\oint_{\ell} \eta=-\left(1-e^{2 \pi i / 3}\right) \int_{0}^{1}\left(a \sqrt[3]{\frac{(1-t)^{2}}{t^{2}(1-a t)}}+\sqrt[3]{\frac{(1-a t)^{2}}{t^{2}(1-t)}}\right) d t \\
\int_{\ell} g^{2} \eta=c^{2}\left(1-e^{-2 \pi i / 3}\right) \int_{0}^{1} \frac{d t}{\sqrt[3]{t(1-t)^{2}(1-a t)^{2}}} .
\end{gathered}
$$

Thus (1-4) is equivalent to

$$
-\int_{0}^{1}\left(a \sqrt[3]{\frac{(1-t)^{2}}{t^{2}(1-a t)}}+\sqrt[3]{\frac{(1-a t)^{2}}{t^{2}(1-t)}}\right) d t=c^{2} \int_{0}^{1} \frac{d t}{\sqrt[3]{t(1-t)^{2}(1-a t)^{2}}}
$$

The right-hand side is clearly positive. So now we estimate the left-hand side:

$$
\begin{aligned}
(\mathrm{LHS}) & =-a \int_{0}^{1} \sqrt[3]{\frac{(1-t)^{2}}{t^{2}(1-a t)}} d t-\int_{0}^{1} \sqrt[3]{\frac{(1-a t)^{2}}{t^{2}(1-t)}} d t \\
& \leq-a \int_{0}^{1} \sqrt[3]{\frac{(1-t)^{2}}{t^{2}}} d t-\int_{0}^{1} \sqrt[3]{\frac{1}{t^{2}(1-t)}} d t \\
& =-a B\left(\frac{1}{3}, \frac{5}{3}\right)-B\left(\frac{1}{3}, \frac{2}{3}\right) \\
& =-\left(\frac{2}{3} a+1\right) B\left(\frac{1}{3}, \frac{2}{3}\right),
\end{aligned}
$$

where $B(x, y)$ is the classical beta function mentioned in Section 2B. Hence, if $-\left(\frac{2}{3} a+1\right) \leq 0,(3-20)$ never holds. That is,

$$
\text { if }-\frac{3}{2} \leq a<0,(3-20) \text { never holds. }
$$


On the other hand, we have

$$
\begin{gathered}
\oint_{\ell^{\prime}} \eta=-\left(1-e^{2 \pi i / 3}\right) \int_{0}^{1}\left(\sqrt[3]{\frac{(t-a)^{2}}{t^{2}(1-t)}}-\sqrt[3]{\frac{(1-t)^{2}}{t^{2}(t-a)}}\right) d t, \\
\int_{\ell^{\prime}} g^{2} \eta=c^{2}\left(1-e^{-2 \pi i / 3}\right) \int_{0}^{1} \frac{d t}{\sqrt[3]{t(1-t)^{2}(t-a)^{2}}}
\end{gathered}
$$

from (3-13). Thus (1-4) is equivalent to

$$
-\int_{0}^{1}\left(\sqrt[3]{\frac{(t-a)^{2}}{t^{2}(1-t)}}-\sqrt[3]{\frac{(1-t)^{2}}{t^{2}(t-a)}}\right) d t=c^{2} \int_{0}^{1} \frac{d t}{\sqrt[3]{t(1-t)^{2}(t-a)^{2}}} .
$$

The right-hand side is again positive. So again we estimate the left-hand side:

$$
\begin{aligned}
(\text { LHS }) & =-\int_{0}^{1} \sqrt[3]{\frac{(t-a)^{2}}{t^{2}(1-t)}} d t+\int_{0}^{1} \sqrt[3]{\frac{(1-t)^{2}}{t^{2}(t-a)}} d t \\
& \leq-\int_{0}^{1} \sqrt[3]{\frac{(-a)^{2}}{t^{2}(1-t)}} d t+\int_{0}^{1} \sqrt[3]{\frac{(1-t)^{2}}{t^{2}(-a)}} d t \\
& =-(-a)^{2 / 3} B\left(\frac{1}{3}, \frac{2}{3}\right)+(-a)^{-1 / 3} B\left(\frac{1}{3}, \frac{5}{3}\right) \\
& =(-a)^{-1 / 3}\left(a+\frac{2}{3}\right) B\left(\frac{1}{3}, \frac{2}{3}\right) .
\end{aligned}
$$

Hence, if $a+\frac{2}{3} \leq 0$, (3-24) never holds. That is,

$$
\text { if } a \leq-\frac{2}{3},(3-24) \text { never holds. }
$$

Combining (3-21) and (3-25), the period condition cannot be solved.

Main Theorem 2 is an immediate consequence of the above arguments.

\section{Remaining problems}

In this section we introduce remaining problems related to this work.

4A. The case that $\gamma$ is odd and greater than 1. For the case that the genus $\gamma>1$ is odd, a complete minimal surface of finite total curvature $f: M=\bar{M}_{\gamma} \backslash\left\{p_{1}, p_{2}\right\} \rightarrow \mathbb{R}^{3}$ which satisfies equality in (1-9) is yet to be found. However, Matthias Weber [2015] has constructed the following examples numerically.

Example 4.1 (Weber). Let $\gamma$ be a positive integer. Define

$$
\begin{gathered}
F_{1}\left(z ; a_{1}, a_{3}, \ldots, a_{2 \gamma-1}\right)=\prod_{i=1}^{\gamma}\left(z-a_{2 i-1}\right), \\
F_{2}\left(z ; a_{2}, a_{4}, \ldots, a_{2 \gamma}\right)=\prod_{i=1}^{\gamma}\left(z-a_{2 i}\right),
\end{gathered}
$$




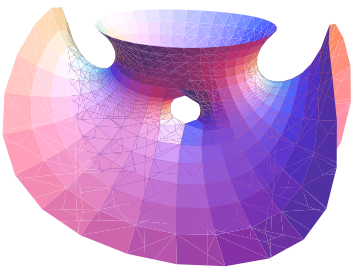

$\gamma=1$

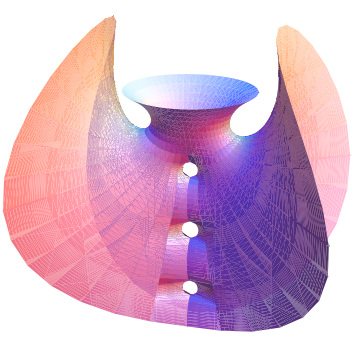

$\gamma=3$

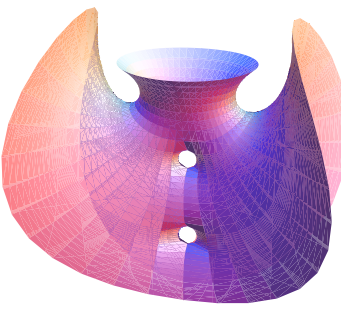

$\gamma=2$

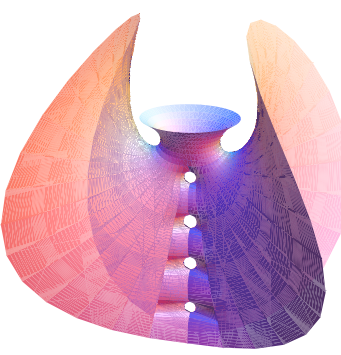

$\gamma=4$

Figure 17. Minimal surfaces of genus $\gamma$ with two ends which satisfy $\operatorname{deg}(g)=\gamma+2$.

where $1=a_{1}<a_{2}<\cdots<a_{2 \gamma}$ are constants to be determined. Define a compact Riemann surface $\bar{M}_{\gamma}$ of genus $\gamma$ by

$$
\bar{M}_{\gamma}=\left\{(z, w) \in(\mathbb{C} \cup\{\infty\})^{2} \mid w^{2}=z \frac{F_{1}\left(z ; a_{1}, a_{3}, \ldots, a_{2 \gamma-1}\right)}{F_{2}\left(z ; a_{2}, a_{4}, \ldots, a_{2 \gamma}\right)}\right\} .
$$

Let $M=\bar{M}_{\gamma} \backslash\{(0,0),(\infty, \infty)\}$. We set

$$
g=c \frac{w}{z+1} \quad \text { for } c>0, \quad \eta=\frac{(z+1)^{2}}{z w} d z .
$$

Then there exist constants $c, a_{2}, a_{3}, \ldots, a_{2 \gamma}$ such that (P) holds. (See Figure 17.)

For $\gamma=1$, we can prove the existence of the surface rigorously. However, for other cases, since the surface does not have enough symmetry, the rigorous proof of the existence still remains an open problem.

4B. Existence of nonorientable minimal surfaces. Our work is devoted to minimal surfaces satisfying $\operatorname{deg}(g)=\gamma+2$. On the other hand, it is important to consider the existence of nonorientable minimal surfaces with $\operatorname{deg}(g)=\gamma+3$. Now, we review nonorientable minimal surfaces in $\mathbb{R}^{3}$.

Let $f^{\prime}: M^{\prime} \rightarrow \mathbb{R}^{3}$ be a minimal immersion of a nonorientable surface into $\mathbb{R}^{3}$. Then the oriented two sheeted covering space $M$ of $M^{\prime}$ naturally inherits a Riemann surface structure and we have a canonical projection $\pi: M \rightarrow M^{\prime}$. We can also 
define a map $I: M \rightarrow M$ such that $\pi \circ I=\pi$, which is an antiholomorphic involution on $M$ without fixed points. Here $M^{\prime}$ can be identified with $M /\langle I\rangle$. In this way, if $f: M \rightarrow \mathbb{R}^{3}$ is a conformal minimal surface and there is an antiholomorphic involution $I: M \rightarrow M$ without fixed points so that $f \circ I=f$, then we can define a nonorientable minimal surface $f^{\prime}: M^{\prime}=M /\langle I\rangle \rightarrow \mathbb{R}^{3}$. Conversely, every nonorientable minimal surface is obtained in this procedure.

Suppose that $f^{\prime}: M^{\prime}=M /\langle I\rangle \rightarrow \mathbb{R}^{3}$ is complete and of finite total curvature. Then we can apply Theorems 1.4 and 1.5 to the conformal minimal immersion $f: M \rightarrow \mathbb{R}^{3}$. Furthermore, we have a stronger restriction on the topology of $M^{\prime}$ or $M$. In fact, Meeks [1981] showed that the Euler characteristic $\chi\left(\bar{M}_{\gamma}\right)$ and $2 \operatorname{deg}(g)$ are congruent modulo 4, where $g$ is the Gauss map of $f$. By these facts, we can observe that for every complete nonorientable minimal surface of finite total curvature, $\operatorname{deg}(g) \geq \gamma+3$ holds.

For $\gamma=0$ and $\gamma=1$, Meeks' Möbius strip [1981] and López's Klein bottle [1993] satisfy $\operatorname{deg}(g)=\gamma+3$. But, for $\gamma \geq 2$, no examples with $\operatorname{deg}(g)=\gamma+3$ are known. So, it is interesting to give a minimal surface satisfying $\operatorname{deg}(g)=\gamma+3$ with an antiholomorphic involution without fixed points. This problem appeared in [López and Martín 1999] and [Martín 2005].

\section{Acknowledgements}

The authors thank Professor Reiko Miyaoka for a fruitful discussion when the authors attended her informal lectures on minimal surfaces at Kyushu University. The authors also thank Professors Shin Kato, Francisco J. López, Wayne Rossman, Masaaki Umehara, Matthias Weber, and Kotaro Yamada for their valuable comments. They would further like to thank the referee for comments that significantly improved the results here.

\section{References}

[Berglund and Rossman 1995] J. Berglund and W. Rossman, "Minimal surfaces with catenoid ends", Pacific J. Math. 171:2 (1995), 353-371. MR 97a:53007 Zbl 1147.53302

[Chen and Gackstatter 1982] C. C. Chen and F. Gackstatter, "Elliptische und hyperelliptische Funktionen und vollständige Minimalflächen vom Enneperschen Typ”, Math. Ann. 259:3 (1982), 359-369. MR 84d:53005 Zbl 0468.53008

[Espírito Santo 1994] N. do Espírito Santo, "Complete minimal surfaces in $\mathbb{R}^{3}$ with type Enneper end”, Ann. Inst. Fourier (Grenoble) 44:2 (1994), 525-557. MR 95h:53008 Zbl 0803.53006

[Fujimori and Shoda 2014] S. Fujimori and T. Shoda, "A family of complete minimal surfaces of finite total curvature with two ends", pp. 19-31 in Differential geometry of submanifolds and its related topics, edited by S. Maeda et al., World Scientific, Hackensack, NJ, 2014. MR 3203469 Zbl 1303.53014 
[Hoffman and Karcher 1997] D. A. Hoffman and H. Karcher, "Complete embedded minimal surfaces of finite total curvature", pp. 5-93 in Geometry, V, edited by R. Osserman and R. V. Gamkrelidze, Encyclopaedia of Mathematical Sciences 90, Springer, Berlin, 1997. MR 98m:53012 Zbl 0890.53001

[Hoffman and Meeks 1990] D. A. Hoffman and W. H. Meeks, III, "Embedded minimal surfaces of finite topology", Ann. of Math. (2) 131:1 (1990), 1-34. MR 91i:53010 Zbl 0695.53004

[Hoffman and Osserman 1980] D. A. Hoffman and R. Osserman, The geometry of the generalized Gauss map, Memoirs of the American Mathematical Society 28:236, American Mathematical Society, Providence, RI, 1980. MR 82b:53012 Zbl 0469.53004

[Huber 1957] A. Huber, "On subharmonic functions and differential geometry in the large", Comment. Math. Helv. 32 (1957), 13-72. MR 20 \#970 Zbl 0080.15001

[Jorge and Meeks 1983] L. P. Jorge and W. H. Meeks, III, “The topology of complete minimal surfaces of finite total Gaussian curvature”, Topology 22:2 (1983), 203-221. MR 84d:53006 Zbl 0517.53008

[López 1992] F. J. López, "The classification of complete minimal surfaces with total curvature

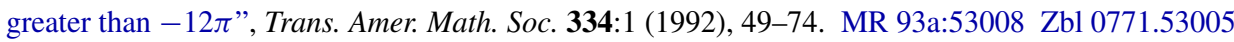
[López 1993] F. J. López, “A complete minimal Klein bottle in $\mathbb{R}^{3}$ ”, Duke Math. J. 71:1 (1993), 23-30. MR 94e:53005 Zbl 0796.53006

[López and Martín 1999] F. J. López and F. Martín, "Complete minimal surfaces in $\mathbb{R}^{3}$ ", Publ. Mat. 43:2 (1999), 341-449. MR 2002c:53010 Zbl 0951.53001

[Martín 2005] F. Martín, "Complete nonorientable minimal surfaces in $\mathbb{R}^{3}$ ”, pp. 371-380 in Global theory of minimal surfaces (Berkeley, 2001), edited by D. A. Hoffman, Clay Mathematics Proceedings 2, American Mathematical Society, Providence, RI, 2005. MR 2006e:53020 Zbl 1106.53006

[Martín and Weber 2001] F. Martín and M. Weber, "On properly embedded minimal surfaces with three ends”, Duke Math. J. 107:3 (2001), 533-559. MR 2002b:53004 Zbl 1044.53006

[Meeks 1981] W. H. Meeks, III, "The classification of complete minimal surfaces in $\mathbb{R}^{3}$ with total

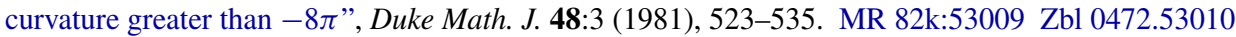

[Meeks and Weber 2007] W. H. Meeks, III and M. Weber, "Bending the helicoid", Math. Ann. 339:4 (2007), 783-798. MR 2008k:53020 Zbl 1156.53011

[Mira 2006] P. Mira, "Complete minimal Möbius strips in $\mathbb{R}^{n}$ and the Björling problem", J. Geom. Phys. 56:9 (2006), 1506-1515. MR 2007d:53012 Zbl 1107.53007

[Nitsche 1975] J. C. C. Nitsche, Vorlesungen über Minimalflächen, Chapter 1-5, pp. 1-430, Grundlehren der mathematischen Wissenschaften 199, Springer, Berlin, 1975. Translated and revised in Lectures on minimal surfaces, 1: Introduction, fundamentals, geometry and basic boundary value problems, Cambridge University Press, 1989. MR 56 \#6533 Zbl 0319.53003

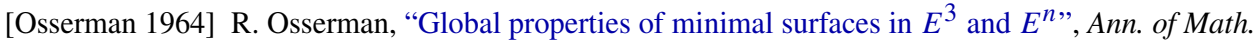
(2) 80 (1964), 340-364. MR 31 \#3946 Zbl 0134.38502

[Sato 1996] K. Sato, "Construction of higher genus minimal surfaces with one end and finite total curvature”, Tohoku Math. J. (2) 48:2 (1996), 229-246. MR 97d:53008 Zbl 1010.53501

[Schoen 1983] R. M. Schoen, "Uniqueness, symmetry, and embeddedness of minimal surfaces", $J$. Differential Geom. 18:4 (1983), 791-809. MR 85f:53011 Zbl 0575.53037

[Weber 2015] M. Weber, Virtual minimal surface museum, 2015, http://www.indiana.edu/ minimal.

[Weber and Wolf 1998] M. Weber and M. Wolf, "Minimal surfaces of least total curvature and moduli spaces of plane polygonal arcs", Geom. Funct. Anal. 8:6 (1998), 1129-1170. MR 99m:53020 Zbl 0954.53007

[Wohlgemuth 1997] M. Wohlgemuth, "Minimal surfaces of higher genus with finite total curvature", Arch. Rational Mech. Anal. 137:1 (1997), 1-25. MR 98j:53015 Zbl 0874.53007 
Received April 1, 2015.

SHOICHI FUJIMORI

DEPARTMENT OF MATHEMATICS

OKAYAMA UNIVERSITY

OKAYAMA 700-8530

JAPAN

fujimori@math.okayama-u.ac.jp

TOSHIHIRO SHODA

FACUlTy OF CUlture AND EduCATION

SAGA UNIVERSITY

1 HONJO-MACHI

SAGA-CITY 840-8502

JAPAN

tshoda@cc.saga-u.ac.jp 


\title{
PACIFIC JOURNAL OF MATHEMATICS
}

\author{
msp.org/pjm
}

Founded in 1951 by E. F. Beckenbach (1906-1982) and F. Wolf (1904-1989)

\section{EDITORS}

Don Blasius (Managing Editor)

Department of Mathematics

University of California

Los Angeles, CA 90095-1555

blasius@math.ucla.edu

\author{
Paul Balmer \\ Department of Mathematics \\ University of California \\ Los Angeles, CA 90095-1555 \\ balmer@math.ucla.edu \\ Robert Finn \\ Department of Mathematics \\ Stanford University \\ Stanford, CA 94305-2125 \\ finn@math.stanford.edu \\ Sorin Popa \\ Department of Mathematics \\ University of California \\ Los Angeles, CA 90095-1555 \\ popa@math.ucla.edu
}

\author{
Vyjayanthi Chari \\ Department of Mathematics \\ University of California \\ Riverside, CA 92521-0135 \\ chari@math.ucr.edu \\ Kefeng Liu \\ Department of Mathematics \\ University of California \\ Los Angeles, CA 90095-1555 \\ liu@math.ucla.edu \\ Jie Qing \\ Department of Mathematics \\ University of California \\ Santa Cruz, CA 95064 \\ qing@ cats.ucsc.edu
}

\section{PRODUCTION}

Silvio Levy, Scientific Editor, production@msp.org

\section{SUPPORTING INSTITUTIONS}

ACADEMIA SINICA, TAIPEI

CALIFORNIA INST. OF TECHNOLOGY

INST. DE MATEMÁTICA PURA E APLICADA

KEIO UNIVERSITY

MATH. SCIENCES RESEARCH INSTITUTE

NEW MEXICO STATE UNIV.

OREGON STATE UNIV.

\author{
STANFORD UNIVERSITY \\ UNIV. OF BRITISH COLUMBIA \\ UNIV. OF CALIFORNIA, BERKELEY \\ UNIV. OF CALIFORNIA, DAVIS \\ UNIV. OF CALIFORNIA, LOS ANGELES \\ UNIV. OF CALIFORNIA, RIVERSIDE \\ UNIV. OF CALIFORNIA, SAN DIEGO \\ UNIV. OF CALIF., SANTA BARBARA
}

\author{
Daryl Cooper \\ Department of Mathematics \\ University of California \\ Santa Barbara, CA 93106-3080 \\ cooper@math.ucsb.edu \\ Jiang-Hua Lu \\ Department of Mathematics \\ The University of Hong Kong \\ Pokfulam Rd., Hong Kong \\ jhlu@maths.hku.hk \\ Paul Yang \\ Department of Mathematics \\ Princeton University \\ Princeton NJ 08544-1000 \\ yang@math.princeton.edu
}

These supporting institutions contribute to the cost of publication of this Journal, but they are not owners or publishers and have no responsibility for its contents or policies.

See inside back cover or msp.org/pjm for submission instructions.

The subscription price for 2016 is US $\$ 440 /$ year for the electronic version, and $\$ 600 /$ year for print and electronic.

Subscriptions, requests for back issues and changes of subscribers address should be sent to Pacific Journal of Mathematics, P.O. Box 4163, Berkeley, CA 94704-0163, U.S.A. The Pacific Journal of Mathematics is indexed by Mathematical Reviews, Zentralblatt MATH, PASCAL CNRS Index, Referativnyi Zhurnal, Current Mathematical Publications and Web of Knowledge (Science Citation Index).

The Pacific Journal of Mathematics (ISSN 0030-8730) at the University of California, c/o Department of Mathematics, 798 Evans Hall \#3840, Berkeley, CA 94720-3840, is published twelve times a year. Periodical rate postage paid at Berkeley, CA 94704, and additional mailing offices. POSTMASTER: send address changes to Pacific Journal of Mathematics, P.O. Box 4163, Berkeley, CA 94704-0163.

PJM peer review and production are managed by EditFLOW ${ }^{\circledR}$ from Mathematical Sciences Publishers.

\section{PUBLISHED BY}

\section{mathematical sciences publishers \\ nonprofit scientific publishing}

http://msp.org/

(C) 2016 Mathematical Sciences Publishers 


\section{PACIFIC JOURNAL OF MATHEMATICS}

Volume 282 No. $1 \quad$ May 2016

On the half-space theorem for minimal surfaces in Heisenberg space

Tristan Alex

Extending smooth cyclic group actions on the Poincaré homology sphere 9

NIMA ANVARI

A short proof of the existence of supercuspidal representations for all reductive $p$-adic groups

RAPHAËL BEUZART-PLESSIS

Quantum groups and generalized circular elements

MICHAEL BRANNAN and KAY KIRKPATRICK

Volumes of Montesinos links

KathleEn Finlinson and Jessica S. PurCell

Minimal surfaces with two ends which have the least total absolute curvature

SHOICHI FUJIMORI and TOSHIHIRO SHODA

Multiplicité du spectre de Steklov sur les surfaces et nombre chromatique

\section{PIERRE JAMMES}

$E$-polynomial of the $\mathrm{SL}(3, \mathbb{C})$-character variety of free groups

SEAN LAWTON and VicEnTE MuÑOZ

The Blum-Hanson property for $\mathscr{C}(K)$ spaces

PASCAL LEFÈVRE and ÉTIENNE MATHERON

Crossed product algebras and direct integral decomposition for Lie supergroups

KARL-HERMANN NEEB and HADI SALMASIAN Associated primes of local cohomology modules over regular rings

TONY J. PUTHENPURAKAL 